\title{
Using a hybrid-fluid model to simulate the ion-hose instability in long-pulse electron linacs
}

\author{
Bruce E. Carlsten \\ Los Alamos National Laboratory, Los Alamos, New Mexico 87545, USA \\ (Received 3 June 2005; revised manuscript received 3 June 2005; published 30 November 2005)
}

\begin{abstract}
A numerical model of the ion-hose instability for long-pulse electron linacs is presented, where the ion motion is represented by fluid parameters. In order to gain extra numerical stability, the fluid behavior of the ions is evolved via particle-in-cell (PIC) techniques. This methodology provides a much faster simulation than a full PIC calculation, allowing for end-to-end simulations of the ion-hose instability in actual linear accelerator configurations. After the description of the simulation model and some simple test cases, the instability is analyzed for a variety of nominal accelerator transport conditions. Simulations of the instability are provided for sections of the DARHT long-pulse accelerator that show different growth regimes of the instability. We find that large-amplitude growth is possible in accelerator and transport regions lacking uniform external focusing, for electron pulse lengths of $2 \mu$ sec and longer.
\end{abstract}

DOI: 10.1103/PhysRevSTAB.8.114202

PACS numbers: 29.27. $-\mathrm{a}$

\section{INTRODUCTION}

The ion-hose instability has been identified as a key limiting factor in long-pulse electron induction linacs [13]. This instability is well known, and has been studied in detail for electron beams in induction linacs operating in the ion-focusing regime (IFR). This instability is similar in function to the resistive hose instability [4,5], and early analyses of it were based on similar theory $[6,7]$. This instability was first experimentally observed in 1986 at the Naval Surface Warfare Center (White Oak) [8] and later at Sandia National Laboratories in 1988 [9], and the growth of this instability had been accurately measured in a recirculating induction accelerator by 1990 [10]. Closely related mechanisms have also been suggested as possible limitations in future linear colliders, known as the fast ionbeam instability $[11,12]$, and observed in storage rings, known there as the $e-p$ instability $[13,14]$.

The ion-hose instability arises when an electron beam passes through the residual gas in a beam line. The electron beam ionizes the gas and can interact with the resulting ions electrostatically. For practical parameters, most, if not all, of the electrons formed during the ionization process (the ionization electrons) are expelled from the location of the primary electron beam and do not cancel the electric field of the ions. If the electron beam's transverse centroid does not vary in time, there is no net force on the centroid from the ions. However, if the beam's centroid varies in time, the relative displacement with the ion distribution will lead to a force deflecting the centroid. If the electron pulse is long enough for several ion oscillations, a parametric resonance can develop and this mutual force can lead to an exponential growth of the centroid offset. The following model shows the basic mechanism.

This model is too simplistic to show many features of the ion-hose instability, and does not include the primary mechanism of the instability used in benchmarking the algorithms in the next section. However, this model shares features with the key mechanism that dominates the instability for long-pulse electron machines. Consider an electron beam traversing a channel that also contains an ion column, offset transversely. The electrons are attracted to the ions as they move axially, and execute betatron oscillations about the centroid of the ions. The ions are assumed to be fixed axially, but are likewise attracted transversely to the electrons. If the ions have infinite mass, the betatron motion of the electrons will be periodic in axial position. However, if the ion mass is sufficiently small (molecular for times scales on the order of $1 \mu \mathrm{sec}$ for kiloAmpere beams), the betatron oscillation amplitude can increase exponentially with distance.

For simplicity, we will assume that both the electron beam and the ion channel have uniform density that is axisymmetric about their transverse centroids, and that the separation between their centroids is small compared to their overall radii. The differential equation governing the motion of the ion centroid $x_{i}(z, t)$ at an axial location $z$ is given by

$$
\frac{d^{2}}{d t^{2}} x_{i}=-\omega_{i}^{2}\left(x_{i}-x_{b}\right)
$$

where $\omega_{i}$ is the ion oscillation frequency and $x_{b}(z, t)$ is the centroid of the slice of the electron pulse at $z$ at time $t$. Likewise, the differential equation for the centroid of the electron slice is given by

$$
\frac{d^{2}}{d z^{2}} x_{b}=-\frac{\omega_{b}^{2}}{v^{2}}\left(x_{b}-x_{i}\right)
$$

where $v$ is the axial beam velocity.

We will look for solutions of the form

$$
x_{b}=\bar{x}_{b} e^{\Omega t+\Gamma z}, \quad x_{i}=\bar{x}_{i} e^{\Omega t+\Gamma z} .
$$

This is not the only possible solution to these equations, but has features relevant to the instability dominating longpulse electron beams. We will be looking for a steady-state 
solution that grows exponentially along the axis. These solutions lead to these equations

$$
\bar{x}_{b} \Gamma^{2}=-\frac{\omega_{b}^{2}}{v^{2}}\left(\bar{x}_{b}-\bar{x}_{i}\right), \quad \bar{x}_{i} \Omega^{2}=-\omega_{i}^{2}\left(\bar{x}_{i}-\bar{x}_{b}\right) .
$$

These equations have four unknowns $-\bar{x}_{b}$ and $\bar{x}_{i}$ are in general complex with an unknown phase relation. Eliminating $\bar{x}_{b}$ and $\bar{x}_{i}$ leads to

$$
0=\Gamma^{2} \Omega^{2}+\frac{\omega_{b}^{2}}{v^{2}} \Omega^{2}+\Gamma^{2} \omega_{i}^{2}
$$

Let us also assume that the beam is injected at $z=0$ with a sinusoidal variation, $\Omega=j \omega$. This leads to the solution

$$
\Gamma^{2}=\frac{\omega^{2} \omega_{b}^{2} / v^{2}}{\omega_{i}^{2}-\omega^{2}},
$$

and the oscillation amplitude will grow exponentially if $\omega<\omega_{i}$, or if the oscillation frequency is less than the ion oscillation period.

We will see similar behavior in detailed numerical simulations of long-pulse electron accelerators. However, additional effects will lead to saturation of the instability which are not included in the above simple analysis. Use of multiple ion species will lead to damping by the mixing of oscillation phases of the different species. Also, as the particle oscillation magnitudes exceed the transverse radii of the beam and ion distributions, the frequency of the oscillations decreases and there is additional phase mixing. For actual accelerators designed for low fractional ionization, the electrons in the beam are confined by an external magnetic field and the oscillations of the ions lead to saturation of the instability as their oscillation amplitude approaches the beam radius.

The ion-hose instability is an important possible limitation to emerging, long-pulse electron induction linacs, such as the 2- $\mu$ sec long DARHT second-axis accelerator [15]. For these types of machines, analytic estimates are insufficient to determine the actual saturated amplitude of the ion-hose instability. Particle-in-cell (PIC) calculations are capable of solving this problem exactly, but simulations over the entire linac, including acceleration and focusing, are too cumbersome to be practical at this point, as moving window frames [16] are irrelevant for long-pulse accelerators, because the electron pulse fills the entire length of the accelerator. Heroic PIC simulations of an accelerator with a length of $50 \mathrm{~m}$ using the code LSP were done in [3], but due to the coarseness of the grid, external focusing and acceleration were smeared and details of the acceleration were lost. The BUCKSHOT code [17] was written to study this instability in the IFR. This code uses a gridless PIC model with an analytic push for the amplitude growth. BUCKSHOT does not include particle acceleration, and even with its limited model, is not capable of modeling a full accelerator design. In this paper, we introduce a new numerical model of this effect that is practical to use for full accelerator designs, complete with acceleration and arbitrary external focusing. It consists of a reduced hybridfluid model for the ion motion, coupled with a slice model for the electron beam, and is capable of calculating the time evolution of the ion-hose instability. The algorithm for simulating this instability in long-pulse electron linacs runs quickly because (1) the fluid model requires the minimum amount of calculations to determine the ion distribution parameters driving the ion-hose instability, (2) the fractional ionization is very small, thus the ion motion only depends on the electric fields from the electron slices and not their own self-fields, and (3) we assume that each electron slice has essentially the same transverse distribution (although with a possible offset), so we can separate the centroid motion from the calculation of the slice transverse distribution. This last approximation was verified during the simulations reported in Refs. $[3,18]$ for parameters of interest for long-pulse accelerators.

We describe the numerical model in detail in the next section. Next, we include simple test cases verifying correct dynamics for both the ions and the electrons. First, the linear motion of the ions in the potential well of a uniform electron beam is found. Next, the linear motion of the electron beam in the potential well of a uniform ion channel is found. The mechanics in the code coupling the ion motion to the electron slice motion is benchmarked against the well-known short-pulse ion-hose instability mechanism in the ion-focusing regime, which has both analytic and numerical solutions.

Following that, we present simulations of the ion-hose instability for various nominal accelerator transport conditions to study the instability growth for the cases of both axially uniform focusing and discrete focusing with short solenoids. We confirm that the growth is suppressed by a continuous axial magnetic field as demonstrated in [3], but additionally see that large-amplitude growth can occur if periodic focusing is used. In the final section, we simulate the ion-hose instability for sections of the DARHT longpulse accelerator that have periodic focusing, using the nominal vacuum specifications but with an alternative focusing tune that encourages ion-hose growth. The ionhose instability is phenomenologically described for both the upstream region near the injector and the downstream region just after the last accelerating induction cell. Considering only a single species of ions $\left(\mathrm{H}_{2} \mathrm{O}^{+}\right)$, largeamplitude growth is found by the end of the $2-\mu \mathrm{sec}$ electron pulse in these regions of periodic external focusing. A larger transported beam size, decreased pressure in the vacuum vessel, and multiple ion species would tend to suppress the growth rate and saturated amplitude.

\section{SIMULATION MODEL}

In this section, we describe the ion-hose instability simulation model. We use a reduced hybrid-fluid model 
for the ion motion in order to simplify the calculation and to reduce the required simulation time for modeling practical accelerator designs.

An exact ion-hose calculation can be made in a straightforward manner using standard PIC techniques. However, the storage and execution time requirements to implement this technique for a full-length long-pulse accelerator design with the required resolution to resolve the beam emittance evolution are prohibitive [3]. The electron beam for a long-pulse accelerator will fill the entire length of the accelerator, on the order of $50 \mathrm{~m}$ for the DARHT long-pulse machine. In addition, this calculation is threedimensional. In order to simplify the simulation technique so a reasonably accurate simulation can be made on a personal computer with short execution times (on the order of an hour on a $2 \mathrm{GHz}$ personal computer), we will make two important assumptions. First, we assume that we can numerically separate the calculation of the transverse centroid motion along the electron-beam pulse from the calculation of the transverse beam distribution. Second, we assume that the influence of the ions' electric field is negligible on their motion (it only contributes collectively to the electron slices' centroids) in comparison to the electric field from the electrons. Also, we use the fact that only the fluid values of the ions are needed to determine the electron centroid motion, and the full ion distribution function does not need to be known.

The induced dipole force (leading to centroid motion) is more sensitive to the ion density than the higher-order radial force (leading to variations in the transverse distribution), thus the first assumption is satisfied for sufficiently small ion densities. It is reasonable to expect that we can separate the effect of the collective ion space charge on the beam slices into orders. The lowest order would be the dipole force, the next order would be axisymmetric focusing (azimuthal mode number $m$ equaling zero), the next order would be a single sinusoidal azimuthal variation in focusing ( $m=1)$, then $m=2$, and so on. For small transverse offsets, as one sees in induction linacs with external focusing, only the first two orders are important. It should be pointed out that the primary purpose for this type of numerical model is to determine accelerator parameters that have unacceptable ionization effects, which include typically relatively small ion-hose amplitudes and emittance growth ratios, so the coupling between orders will be small. The lowest order will be described in detail in this paper, and the $m=0$ order has been recently described for long-pulse electron linacs [19]. This is important, since we only need to resolve the electric field from the ions at the location of the electron slice centroids (which is a onedimensional calculation), and not everywhere (which is a two-dimensional calculation, and much slower). Also, the type of electron beams described here are all space-charge dominated, and thus minor emittance growths will not have appreciable effects on the electron-beam slice distribution.
The second assumption is clearly valid for fractional ionizations on the order of $10^{-3}$ and below. Finally, use of the fluid model can be justified by considering the moments of the Vlasov equation [20]. The zero order moment leads to the equation of continuity,

$$
\frac{\partial n}{\partial t}+\vec{\nabla} \cdot(n \vec{u})=0
$$

and the first order moment leads to the fluid equation of motion,

$$
m\left[\frac{\partial \vec{u}}{\partial t}+(\vec{u} \cdot \vec{\nabla}) \vec{u}\right]=e(\vec{E}+\vec{u} \times \vec{B}),
$$

where $n$ is the real-space density function found from the distribution function by

$$
n=\int f(\vec{r}, \vec{v}) d \vec{v} .
$$

Likewise,

$$
\vec{u}=\int \vec{v} f(\vec{r}, \vec{v}) d \vec{v}
$$

is the average velocity at a point $\vec{r}$ in real space. These fluid equations are exact moments of the Vlasov equation, and since the ion space-charge field only depends on $n$ (their velocities are too small to create a significant magnetic field), the fluid equations are sufficient to fully describe the evolution of the ion space-charge field. This is an important result, because it relieves us from explicitly knowing the full ion distribution function and greatly simplifies the numerical calculation.

One further step is made in the simulations reported here. An instantaneous PIC model is used for finding the evolution of the density and the average velocity instead of directly evolving the continuity equation [Eq. (7)] and the fluid equation for the flow of momentum [Eq. (8)] on a finite-difference grid. This approach is still consistentthe importance of Eqs. (7) and (8) is that they show that only density and average velocity components are needed to evolve the density accurately, and that indeed any two distributions with the same discretized real-space densities and average velocities will evolve the same. This is a powerful concept, because it allows us to substitute any specific distribution function at any time, as long as it has the same density and average velocity profiles as the original distribution function. So at each time step, we will substitute a simple PIC distribution model with particles at the grid intersection points for the distribution function, which allows us to numerically evolve the distribution in an easy and straightforward manner. After evolving this distribution, new density and average velocity components are found on the grid, providing the parameters for the next time step. It should be pointed out that because the fluid equations fully describe the beam density, 
the space-charge fields to any order in $m$ can be found in this manner.

Using these assumptions, we can create a simulation model of an accelerator including features required for including the ion-hose instability. We will numerically model the long-pulse electron beam as a series of short slices. We will evolve the transverse distribution of the first slice, and assume the transverse distribution of all other slices are the same. We will follow the centroid of each slice individually. Numerically, this is done by stepping the beam axially, and then evolving the interaction between the ions and the electron-beam slices at that axial location, finding the centroid of each electron slice and the ion distribution as a function of time as the rest of the beam passes that axial location.

An additional important consideration for using this procedure is understanding the behavior of the ionized electrons in the presence of an external axial magnetic field that may be present in order to confine the electron beam. This field has the potential of also restricting the electrons' transport to the beam pipe wall, and may decrease the net ionization. We can study the motion of the ionized electrons by numerically integrating their nonrelativistic equation of motion

$$
m \frac{d}{d t}(\dot{x}, \dot{y}, \dot{z})=\frac{e I}{2 \pi c \varepsilon_{0} a^{2}}(x, y, 0)+e(\dot{x}, \dot{y}, \dot{z}) \times\left(0,0, B_{0}\right)
$$

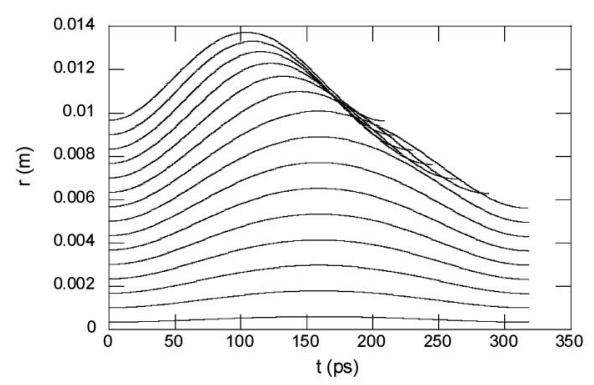

(a)

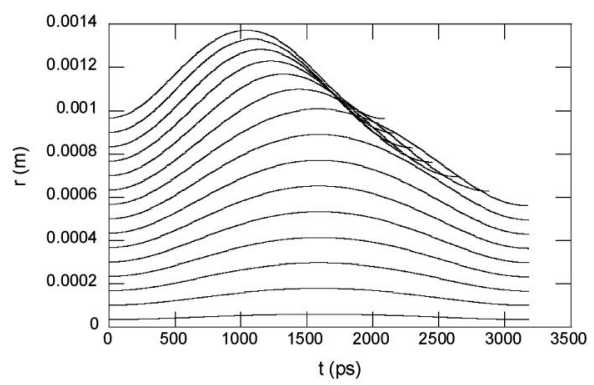

(c) inside the electron beam and

$$
\begin{aligned}
m \frac{d}{d t}(\dot{x}, \dot{y}, \dot{z})= & \frac{e I}{2 \pi c \varepsilon_{0}\left(x^{2}+y^{2}\right)}(x, y, 0)+e(\dot{x}, \dot{y}, \dot{z}) \\
& \times\left(0,0, B_{0}\right)
\end{aligned}
$$

outside the beam, where $I$ is the beam current, $a$ is the beam edge radius, and $B_{0}$ is the applied external axial field, for a uniform density, space-charge dominated beam. Inspection of these equations of motion show that the normalized trajectories $r / a$ scale as $I /\left(a^{2} B_{0}^{2}\right)$. To verify this scaling, we plot in Fig. 1 trajectories for ionized electrons, created at 15 evenly spaced positions in the drive beam, for a beam current, external field, and beam radius of (a) $2 \mathrm{kA}, 0.2 \mathrm{~T}, 1 \mathrm{~cm}$, (b) $20 \mathrm{~A}, 0.2 \mathrm{~T}, 1 \mathrm{~mm}$, and (c) $0.2 \mathrm{~A}$, $0.02 \mathrm{~T}, 1 \mathrm{~mm}$, respectively. All cases have the same value of $I /\left(a^{2} B_{0}^{2}\right)$, and show the same normalized trajectories. The trajectories are plotted for one cyclotron period, which varies for the different trajectories and between cases. Note that the cyclotron periods are short relative to long-pulse electron beams ( $\mu$ sec time scales), and since the ionized particles are created continuously, we can consider the density of these trajectories in a time-averaged sense. Averaged over time, $48 \%$ of the ionized electrons are outside the drive beam for all three cases, so we can assume that the effective ionization of the gas is only $52 \%$ of the total ionization. (This is somewhat of an underestimate because additionally as the ion channel moves transversely,

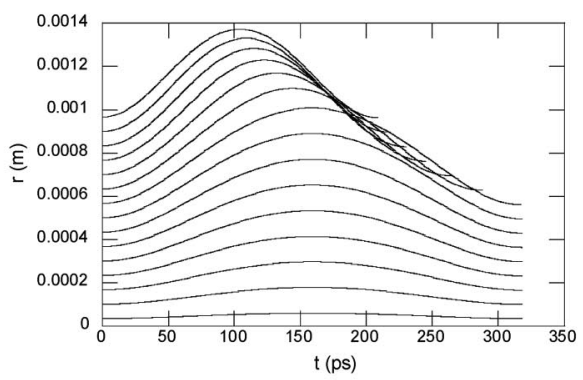

(b)

FIG. 1. One cyclotron orbit of electrons originated at 15 evenly spaced different initial locations within the primary electron beam for the parameters of (a) beam current $2 \mathrm{kA}$, focusing field $0.2 \mathrm{~T}$, beam radius $1 \mathrm{~cm}$, (b) beam current $20 \mathrm{~A}$, focusing field $0.2 \mathrm{~T}$, beam radius $1 \mathrm{~mm}$, and (c) beam current $0.2 \mathrm{~A}$, focusing field $0.02 \mathrm{~T}$, beam radius $1 \mathrm{~mm}$. On average, $48 \%$ of the current is outside the beam. 
more of the ionization electrons will be lost, but this is a good estimate for small ion centroid displacements.)

In Fig. 2, we plot the fraction of the ionization electrons outside the uniform density, space-charge limited beam, as a function of the scaling parameter $I /\left(a^{2} B_{0}^{2}\right)$. As the field vanishes (and the ionization electrons are not bound), the fraction of ionization electrons that stay within the primary beam vanishes, as expected. For values of $I /\left(a^{2} B_{0}^{2}\right)$ greater than 10 , the error in the net ionization from simply using the ionization rate equation is $10 \%$ or less. The final assumption of this model is that the scaling parameter $I /\left(a^{2} B_{0}^{2}\right)$ is relatively large (at least a few, and typically greater than ten), which is valid for typical cases of interest, which we will verify for the test cases.

\section{A. Specific simulation mechanism}

The accelerator simulations were performed by the code SLICE [21]. The SLICE code self-consistently pushes particles in a single axial slice of the beam using the Lorentz force equation, including the beam's self-radial electric, axial magnetic, and azimuthal magnetic fields and all external fields to fourth order in radius. The code uses the long-beam approximation, in which Gauss's law is used for the radial electric field and Ampere's law is used for the azimuthal and axial magnetic fields. A collection of particles (typically 4000 in these simulations) is used to describe the beam at each axial position. As a group, these particles are stepped axially, with a $1 \mathrm{~mm}$ step size.

With the separation assumption, the transverse distribution only needs to be calculated for one axial slice of the beam pulse, but the centroid position needs to be calculated along the entire beam pulse at every axial location. To do this, the electron-beam pulse is divided into separate slices. The total transverse distribution is calculated for the first slice, and is assumed to be the same (except for centroid position) for all the following slices. Ions are produced

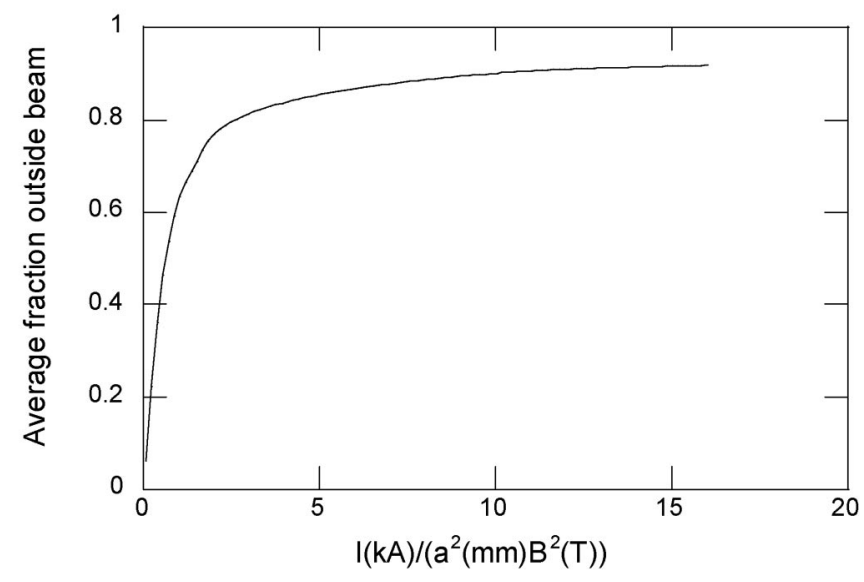

FIG. 2. Fraction of ionization electrons outside primary beam, as a function of the scaling parameter $I /\left(a^{2} B_{0}^{2}\right)$. from each slice from primary impact ionization, given by the rate equation [22]:

$$
\frac{d n_{i}}{d t}=n_{g} n_{e} \sigma v
$$

where $n_{i}$ is the ion density, $n_{g}$ is the initial gas density (assumed to be uniform over radial distances), $n_{e}$ is the electron bunch density (which is a function of time at a given axial and radial position), $\sigma$ is the cross section, and $v$ is the velocity of the electron beam (which we will assume to be the speed of light, $c$ ). Numerically, the cross section can be modified to account for a loss of ionization caused by a relatively small scaling factor $I /\left(a^{2} B_{0}^{2}\right)$. For an electron-beam slice of temporal length $\tau$, the increase in the fractional ionization is then given by

$$
\frac{\Delta n_{i}}{n_{e}}=n_{g} \sigma v \tau=\sigma \tau(\mathrm{sec}) P_{g}(\text { torr }) 9.783\left(10^{26}\right) \mathrm{cm}^{-2}
$$

where $P_{g}$ is the gas pressure in torr.

The numerical procedure is to first step the beam axially, and then to perform the ion-related calculations for all beam slices at that axial location. The ions generated by primary impact ionization are assumed to be axially fixed, but transversely mobile. The ion distribution is calculated after each slice, on a transverse grid of typical size 51 by 51 grid lines. Only the ion density and average velocity is kept at each grid intersection point, as these parameters fully define the ion fluid. For the first slice, there is no ambiguity. Ion density is assigned to each node in accordance with the rate equation [Eq. (13)] and the local electron-beam density. An ion velocity of zero is assigned to each grid intersection point. Next, the force on the slice centroid from this ion density is calculated. (For the first slice, there is no net dipole force as SLICE assumes each slice is axisymmetric about its centroid position.) Finally, the ion distribution is evolved under the influence of that electronbeam slice's electric field. For the ions just from the first slice, this force is axisymmetric, and the ion centroid does not move. The fluid density and velocity can be evolved using standard finite-difference techniques, using the convective form of the Lorentz force law [Eq. (8)] to evolve the fluid velocity and the continuity equation [Eq. (7)] to evolve the fluid density. However, the electron-beam density is often hard edged, leading to a discontinuity in the ion distribution. In order to add extra stability, the fluid model parameters are evolved in a PIC manner instead, as was described above. At each grid intersection point, we assign a superion with the total ion density corresponding to all the ion density within a grid square centered at that intersection point, and with velocity equal to the fluid velocity at that point. We let these superions move for the time period corresponding to the slice length, and afterwards assign new ion densities and average velocities to the grid intersection points. This procedure is then 
followed for all the successive electron pulse slices. For the additional ionization due to new electron slices, additional ion density is assigned to the grid intersection points while keeping the total ion momentum constant. For successive slices, the ion and slice centroids will diverge, leading to both slice and ion dipole forces.

This procedure greatly simplifies the calculation. Because each electron slice itself stays axisymmetric about its centroid, the resulting force on each ion is simple to find (it is essentially a one-dimensional calculation and scales linearly with the number of electrons followed in the simulation). Though the ion distribution can become very distorted, the calculation of the force on only the electron slice centroids also scales linearly with the number of grid intersection points, and is likewise very fast. With the fluid model for the ions, the number of ions that are needed to use in both the ion evolution step and the slice centroid evolution step is equal to the total number of grid intersection points, and never increases. Without the fluid model, the number of ions followed would linearly increase with the slices, and this calculation would become prohibitively slow. Likewise, without the assumption of separating the electron transverse distribution from the centroid positions, calculating the ion motion would require a complex two-dimensional field solver, with execution time scaling more than linear with the number of electrons followed in the simulation.

Another important assumption is that the secondary electrons do not appreciably effect the ionization. This is not obvious, because they are transversely accelerated by the primary beam up to (and then beyond) the peak value of the ionization cross section as a function of electron energy. To estimate this effect, we use the ionization formula [Eq. (13)], with a nominal maximum cross section of $3 \times$ $\left(10^{-20}\right) \mathrm{m}^{2}$ (see, for example, Ref. [23] for argon) at an energy of $100 \mathrm{eV}$ (velocity of $0.02 \mathrm{c}$ ), and a time of $1 \mu \mathrm{sec}$. Note that the electron density $n_{e}$ is equal to the ion density $n_{i}$ times a coupling factor $\kappa$, which cannot exceed unity (and which is typically about 0.1 from Fig. 1). This leads to a fractional increase in the ionization from secondary impact of about $\Delta n_{i} / n_{i}=\kappa 5.6\left(10^{-4}\right)$ over a time of $1 \mu \mathrm{sec}$, which is negligible. Note that even if the secondary electron velocity is increased to $c$, the fractional ionization increase is about $2 \%$, even neglecting the drop in ionization cross section at higher electron energy. We can conclude that the secondary ionization from the secondary electrons is negligible.

Comparing the speed of the hybrid calculation with a mulitparticle PIC simulation, the hybrid model can simulate a 50-m long accelerator with a $2 \mu \mathrm{sec}$-long electron with an axial resolution of $1 \mathrm{~mm}$ in about an hour, whereas a mulitparticle PIC simulation requires on the order of $10 \mathrm{~h}$ for an equivalent geometry (including acceleration and a realistic magnetic field profile), but with only a $5-\mathrm{cm}$ axial resolution. Overall with similar resolution, the hybrid model yields a calculation well over 2 orders of magnitude faster. The cost in accuracy of this faster calculation is the approximation that all electron slices in the primary beam have the same transverse distribution (although potentially offset), but which has been verified to be a minor effect for the types of simulations we are interested [18].

\section{TEST CASES}

At this point it is important to simulate some simple test cases to verify the operation of this ion-hose model. The two algorithms that we want to validate are the centroid evolution algorithm and the ion evolution algorithm. For these tests, we start with a $60 \mathrm{nsec}$ electron pulse separated into 30 slices of $2 \mathrm{nsec}$ apiece. We assume that the centroids of all slices are offset only along the vertical axis, in a half sinusoid, shown in Fig. 3. The maximum vertical offset is $1 \mathrm{~cm}$. The electron-beam current is $4 \mathrm{kA}$, with an edge radius of $3 \mathrm{~cm}$. The magnetic field required to obtain balanced flow at $2 \mathrm{MeV}$ is about $0.037 \mathrm{~T}$. We assign an initial horizontal velocity to the centroids given by

$$
v_{x, \text { cent }}=\frac{y_{\text {cent }} e B}{m \gamma}
$$

which will balance the focusing magnetic force with the centrifugal force. The evolution of the horizontal and vertical centroid positions is shown in Fig. 4, and the total radius of the centroids is shown in Fig. 5, as a function of axial position, which is numerically stable (the ripple is due to a slight mismatch in the applied magnetic field).

The next test case will be for the ion density oscillating in the potential well of the electron distribution. For this case, the electron slice centroids are assumed to be perfectly aligned, and an initial ion distribution is established offset $1 \mathrm{~cm}$ vertically. The ionization process is turned off so we can see the ion centroid evolution in the field from

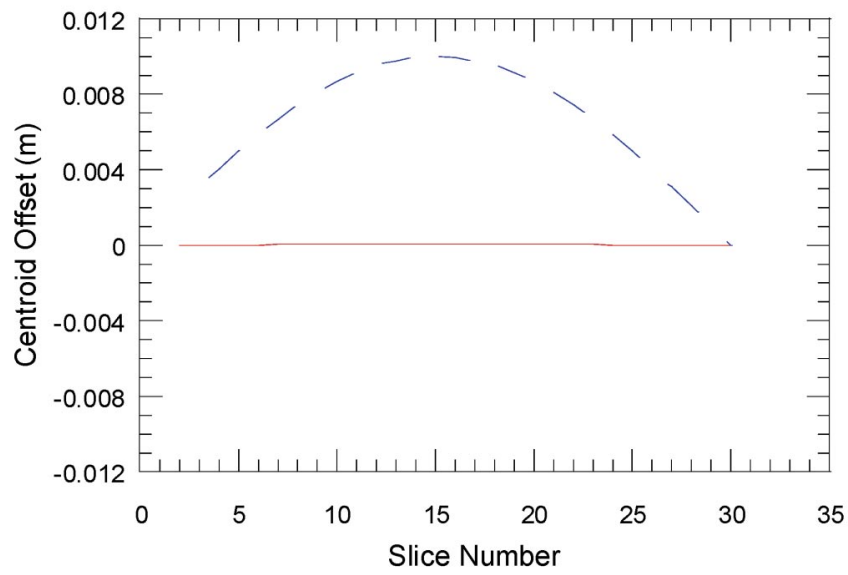

FIG. 3. (Color) Initial electron-beam centroid offsets, versus slice number. The red line is the horizontal offset and the blue line is the vertical offset. 


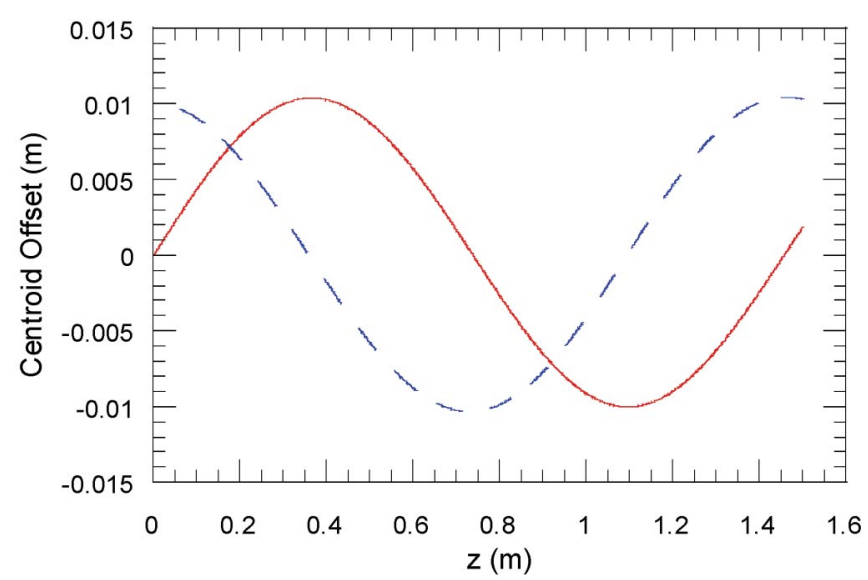

FIG. 4. (Color) Electron-beam centroid position for corkscrewing case. The red line is the horizontal offset and the blue line is the vertical offset.

the electron beam. We see the ion centroid does execute sinusoidal motion in Fig. 6.

The transverse ion motion obeys this harmonic equation

$$
\ddot{x}_{\text {ion }}=-x_{\text {ion }} \frac{e I}{m_{i} 2 \varepsilon_{0} c \pi a^{2}},
$$

which, for these parameters, leads to an oscillation period of $40 \mathrm{nsec}$ for hydrogen ions. With $2 \mathrm{nsec}$ per slice, this is in excellent agreement with Fig. 6.

The next test case we will look at is the evolution of an electron slice centroid initially offset $1 \mathrm{~cm}$ horizontally, in a uniform ion channel centered about the origin. The centroid should obey this equation

$$
\ddot{x}_{\text {cent }}=-x_{\text {cent }} \frac{e I}{m_{e} \gamma 2 \varepsilon_{0} c \pi a^{2}} \sigma c n_{g} \tau,
$$

where the ion channel was created by primary impact ionization from a slice of the beam of length $\tau$ into a

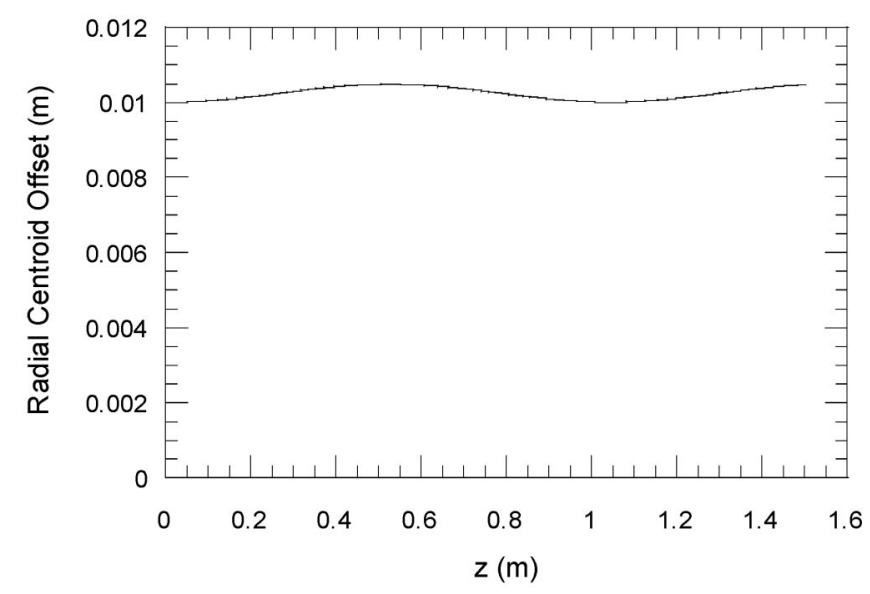

FIG. 5. . Electron centroid position for corkscrewing case, showing total radial offset.

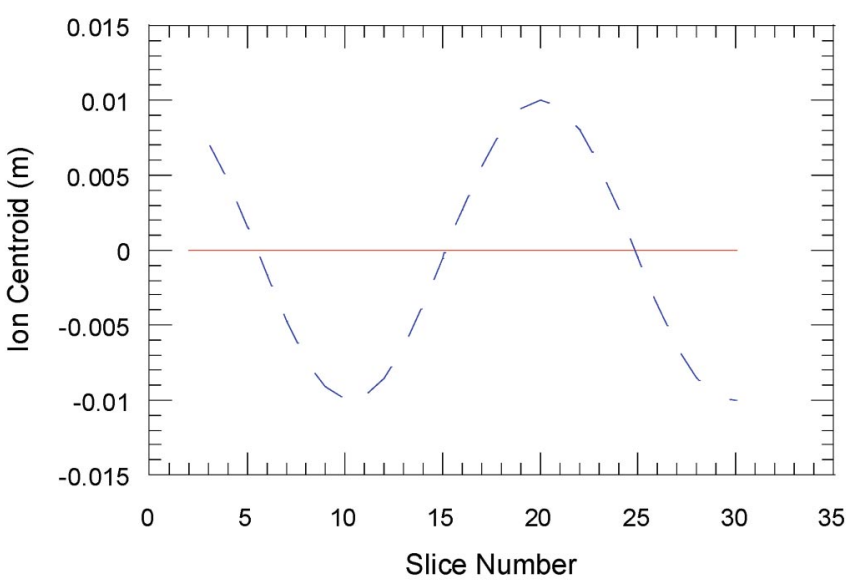

FIG. 6. (Color) Ion centroid motion in the electron beam's potential well. The red line is the horizontal offset and the blue line is the vertical offset.

residual gas. For a slice beam current of $4 \mathrm{~mA}$ (in order to turn off the focusing magnetic field) and length $2 \mathrm{nsec}$, and a pressure of $10^{-7}$ torr and cross section of $8 \times$ $10^{-9} \mathrm{~m}^{2}$, this leads to a betatron wavelength of $1.54 \mathrm{~m}$, again in excellent agreement with the calculation, shown in Fig. 7. It should be pointed out that an absurdly high cross section was needed in order to get a reasonable betatron period for the case of such a low current and short ionization time.

The final test case we will present will be for short-pulse, high-fractional ionization. This instability has been studied in great detail for short-pulse transport in the ion-focusing regime (IFR) [22,24]. The instability has an analytic description in this regime and several analytic and numerical checks can be made. We will benchmark our routine against the simplest analytic test case, that of an electron-beam offset to an initially preionized channel, with equal charge density as that of the beam. The electron

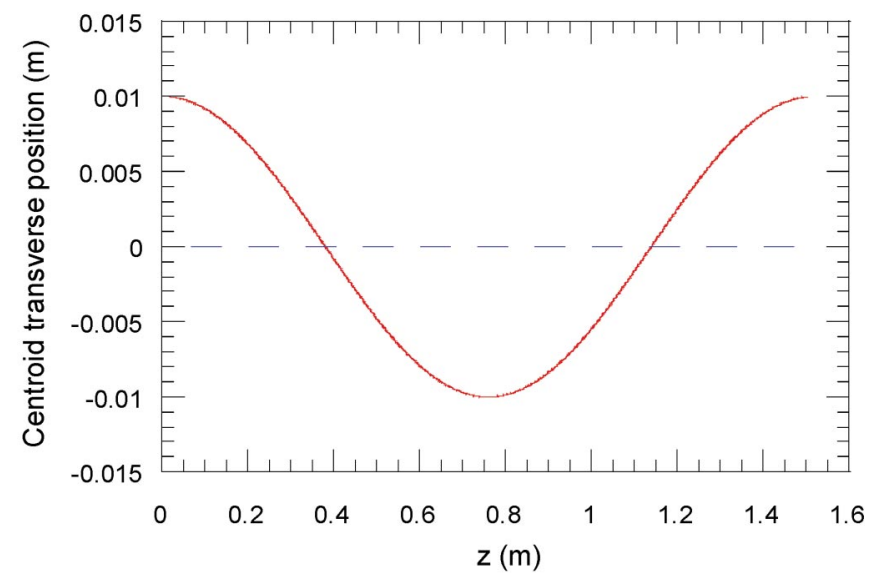

FIG. 7. (Color) Electron-beam slice centroid betatron motion in an ion channel. The red line is the horizontal offset and the blue line is the vertical offset. 
beam and ion channel are of uniform density and of equal radii, and the offset is a small fraction of the beam and ion channel radii.

The initial electron slices in the beam will oscillate in the ion channel with simple betatron motion (as shown in Fig. 7), because the relatively heavy ions will take time to move. Since all electron slices follow nearly the same path, the ions at each axial location will be accelerated with essentially uniform force toward the betatron oscillation position at that axial location. The deformed ion channel will have the same betatron period as the slice centroid, with lesser amplitude.

For this case, we will assume that the fractional ionization is unity. The following simple model will describe the key instability mechanism for this regime. The net force on the electron slice centroid obeys this second-order differential equation:

$$
\ddot{x}_{b}=\frac{e I}{m_{e} \gamma 2 \varepsilon_{0} c \pi a^{2}}\left(x_{i}-x_{b}\right) .
$$

With the assumption that $x_{i}=\varepsilon x_{b}$ where $\varepsilon$ is a (complex) scalar, this has solution

$$
x_{b}=x_{0} e^{j k(1-\varepsilon)^{1 / 2} z},
$$

where $k^{2}=\frac{e I}{m_{e} \gamma 2 \varepsilon_{0} c \pi a^{2}}$.

Note that this solution has a longer betatron period than without the ion motion, since $\varepsilon$ is mostly positive real.

Because of inertia, the ion channel cannot increase its oscillation period as fast as the electron slices, and the effective ion oscillation period is shorter than that for the slices. This implies

$$
\varepsilon=\varepsilon_{r}+j \varepsilon_{i},
$$

where $\varepsilon_{r}$ and $\varepsilon_{i}$ are both real and positive. Expanding the solution for the slice centroid, we find

$$
x_{b}=x_{0} e^{j k\left(1-\varepsilon_{r}\right)^{1 / 2} z} e^{k \varepsilon_{i} z / 2\left(1-\varepsilon_{r}\right)} .
$$

Since $\varepsilon_{i}$ is real, there is exponential growth in the magnitude of the betatron oscillations.

There are two key elements leading to this amplitude growth. First, the ion centroid oscillation increases the electron slice centroid betatron period. Once the electron slices fall behind in phase relative to the ion centroids, the amplitude of their betatron oscillations increases exponentially.

Because of the large electric field from the ions (full fractional ionization), this effect can only be modeled by SLICE with an additional feature added. With full fractional ionization, both the electrons and ions are mostly in electrostatic equilibrium relative to their rms radii. For the electrons, we simulate this by only doing a onedimensional push (in the axial direction), ensuring that the original transverse electron distribution is kept. Calculating the true electrostatic self-fields from the ions is two-dimensional, and very time consuming. Instead, we assume that the ion distribution is axisymmetric and uniform about its centroid, and add in an analytic approximation for its own self-field based only on the actual ion distribution rms radius. In the following simulations, the first electron slice will provide an ionized channel centered about $x=2 \mathrm{~mm}$, with no additional ionization from successive electron slices. All successive electron slices will be injected on axis. The 1-kA electron beam is $2 \mathrm{~cm}$ in radius, with an energy of $3.6 \mathrm{MeV}$. The nominal ion mass used these calculations is $1 / 10$ that of helium (in order to reduce the overall evolution time required in these simulations). Specifically, two methods for determining the centroid motion with fractional ionization will be employed. In both methods, the ions' self-fields will be included with the analytic form described above for the electric field from an axisymmetric and uniform distribution. To check the validity of this approximation, the force on the electron slices' centroids will be found by (1) assuming the same analytic form for the electric field from the ion distribution and comparing that to (2) the electric field found by exact grid calculation. This comparison is a good check on the analytic form, since both methods should give essentially the same result.

Without introducing the additional electric field from the ion themselves, the ion distribution focuses over time. In Fig. 8 we see this effect, over the first $10 \mathrm{nsec}$ of the beam (each electron slice corresponds to $1 / 3 \mathrm{nsec}$ ). Note that without the fractional ionization, the ion rms radius decreases at exactly the same rate that the centroid moves, as it should. For this simulation, the grid spacing is $0.16 \mathrm{~mm}$ and the beam radius crosses two grid lines as it compresses. Although this simulation is nonphysical because it does not include the fractional ionization, it is useful since it demonstrates that the rms radius is being modeled very accu-

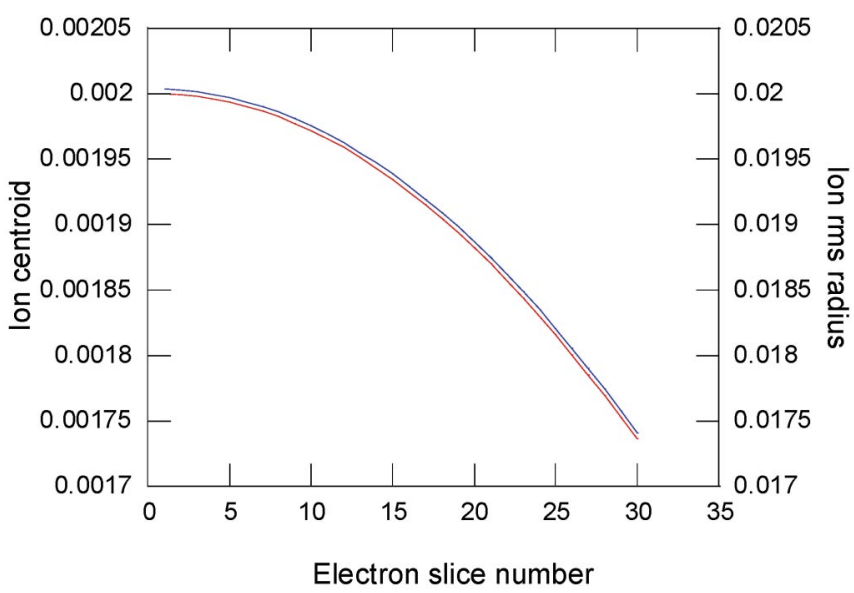

FIG. 8. (Color) Evolution of both the ion centroid and rms radius, for the test case using only the electrons' space charge to check the ion distribution calculation. The red line is for the ion centroid and the blue line is for the ion rms radius. 


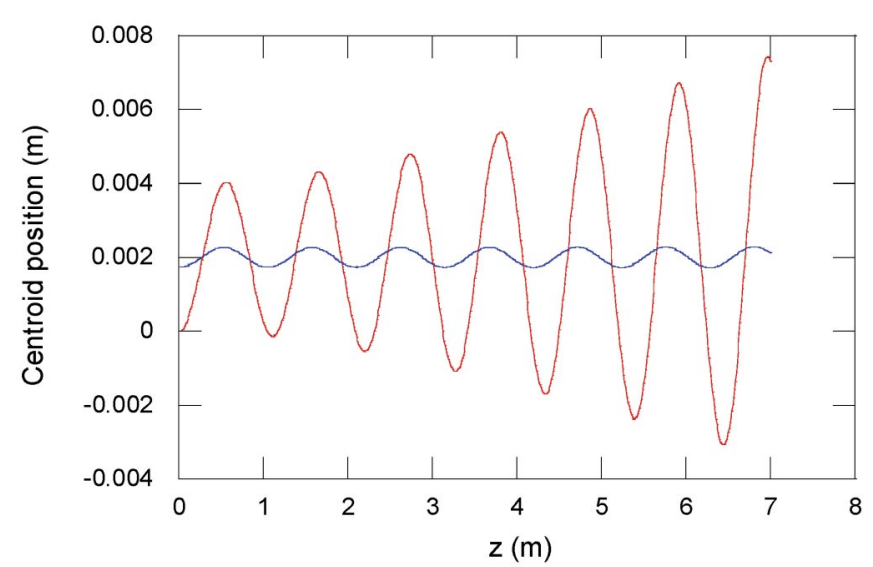

FIG. 9. (Color) Ion-hose calculation in the IFR using method 1. The red line shows the electron slice centroid and the blue line shows the ion centroid.

rately with the fluid model, even as particles are clearly crossing through grid intersection points.

With the fractional ionization included by method 1 , the electron slice and corresponding ion distribution centroid at $10 \mathrm{nsec}$ into the pulse as a function of axial location is shown in Fig. 9. The electric field inside the ion distribution is assumed to be $E_{r}=-\operatorname{Ir} / 2 \varepsilon_{0} \pi c \beta b^{2}$, where $b$ is the ion distribution rms radius. The electron centroid is oscillating about the ion column centered at $x=2 \mathrm{~cm}$, and the ion distribution is executing smaller oscillations. The electron oscillation betatron wavelength has increased due to the ion oscillations as predicted, and there is a corresponding exponential growth in the electron centroid oscillation amplitude. The growth rate is very close to the analytic solution [24].

In Fig. 10 we compare the horizontal electric field calculated by the fluid model to that found from the ana-

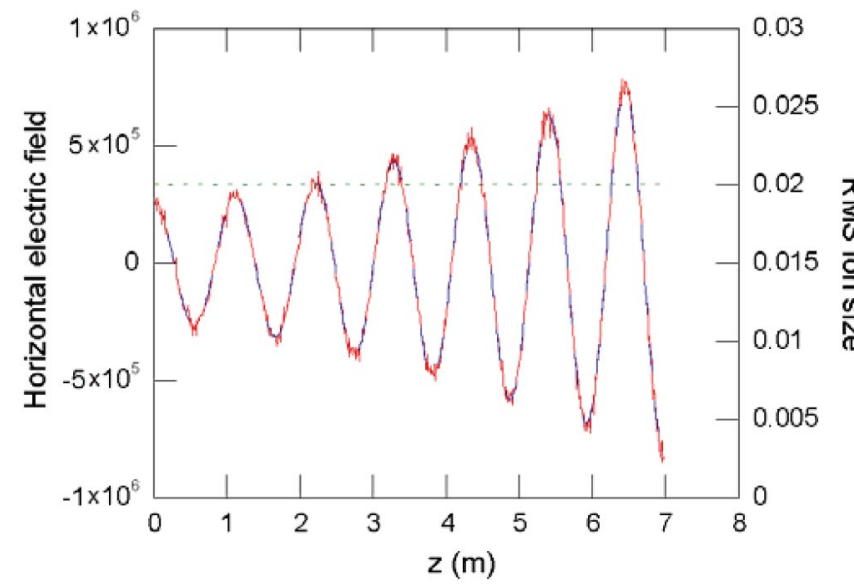

FIG. 10. (Color) Comparison of the electron centroid grid and analytic electric fields, using method 1 . The solid red line shows the electric field calculated on the simulation grid, the dashed blue line shows the electric field calculated by the analytic model, and the dashed green line shows the rms ion size.

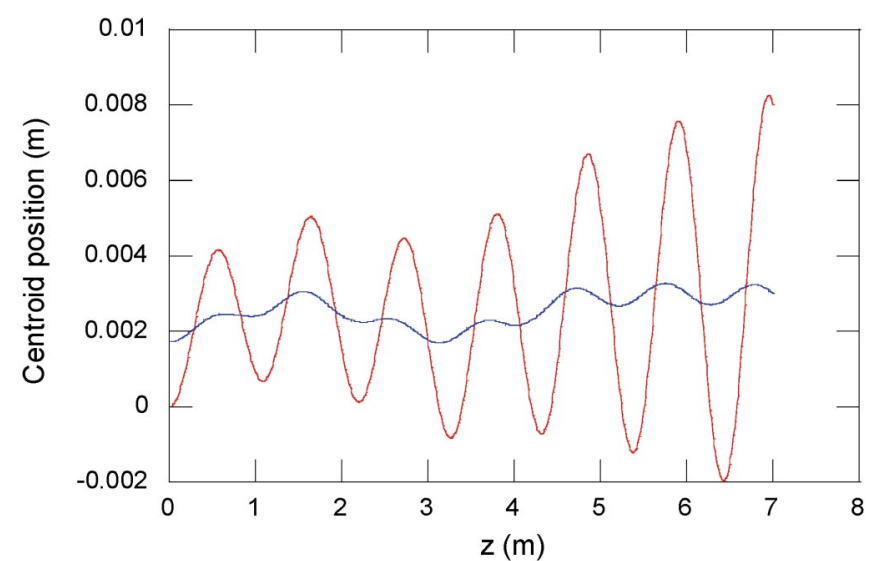

FIG. 11. (Color) Ion-hose calculation in the IFR using method 2. The red line shows the electron slice centroid and the blue line shows the ion centroid.

lytic solution for a uniform distribution, that the electron slice centroid sees. The small hash is noise from the grid, but the overall agreement is also very good. In addition, the growth rate is in good agreement with analytic estimates [22,24].

In the next two figures, Figs. 11 and 12, we redo the calculation using method 2 to calculate the electron centroid motion. Here, the analytic form is used to describe the ions' self-fields, but an exact grid calculation is used for the electron slice centroids. Essentially the same results occur, however there is some additional oscillations in both the ions' and electron slice's centroids. This oscillation is due to the long-term transverse plasma oscillations of the ions (leading to slight periodic nonuniformities in the distribution), but does not appreciably change the results. Because these plasma oscillations are physical, method 2 is also

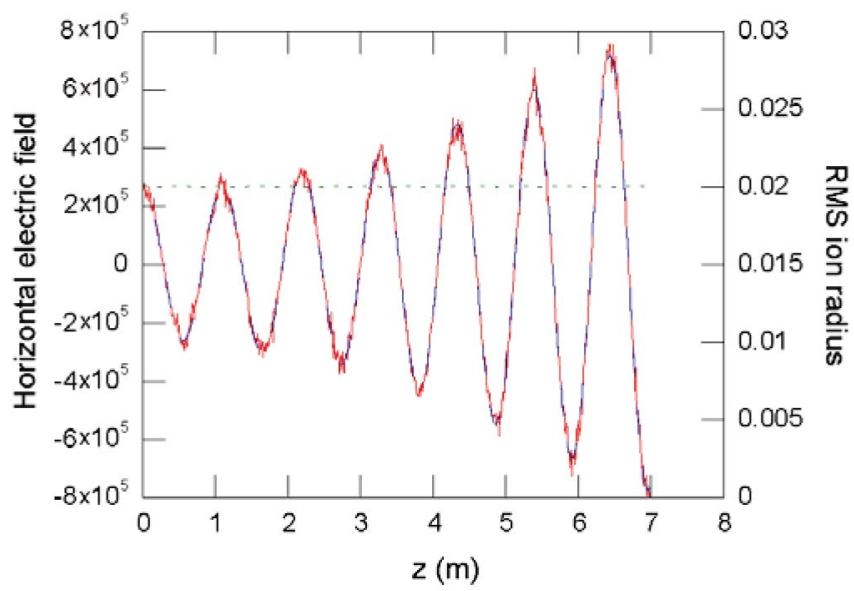

FIG. 12. (Color) Comparison of the electron centroid grid and analytic electric fields, using method 2 . The solid red line shows the electric field calculated on the simulation grid, the dashed blue line shows the electric field calculated by the analytic model, and the dashed green line shows the rms ion size. 


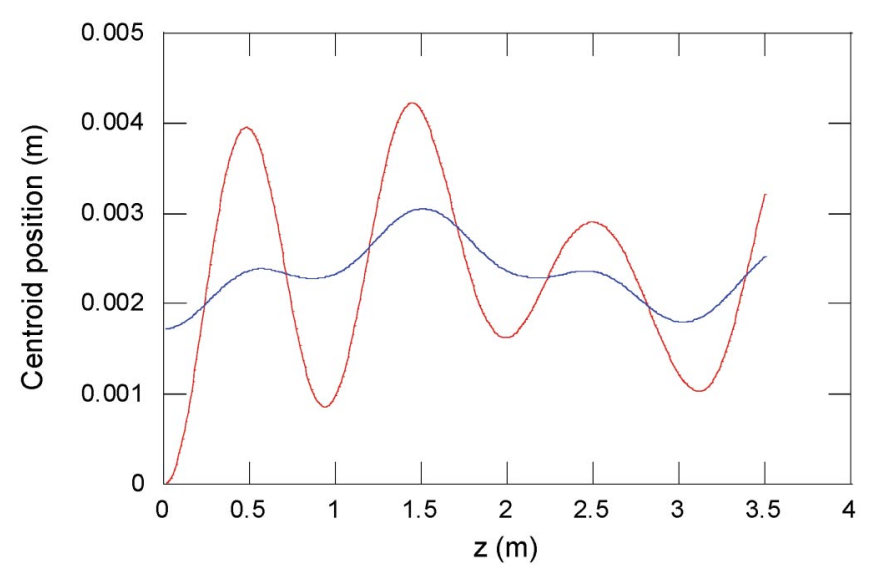

FIG. 13. (Color) Ion-hose calculation in the IFR using method 2, without fractional ionization. The red line shows the electron slice centroid and the blue line shows the ion centroid.

considered to be an accurate way of representing fractional ionization for simulations in this regime.

The previous figures provide some important physical insight into the mechanism. The key feature leading to the instability growth is the increase in the electron's betatron period. However, without full fractional ionization, the rms ion distribution radius decreases (Fig. 8), and in fact the electron's betatron period will decrease. This phase relation between the electron and ion oscillations will lead to attenuation of the electron centroid oscillation amplitude, as predicted in Eq. (21). In Figs. 13 and 14 we show the ion and electron centroids and the calculated electric fields, for the case where the fractional ionization is not included. We see the attenuation of the oscillations, and also the same centroid wandering as seen in Fig. 11, due to plasma oscillations in the ion distribution. For this case, the increased electric field as the ion distribution pinches ac-

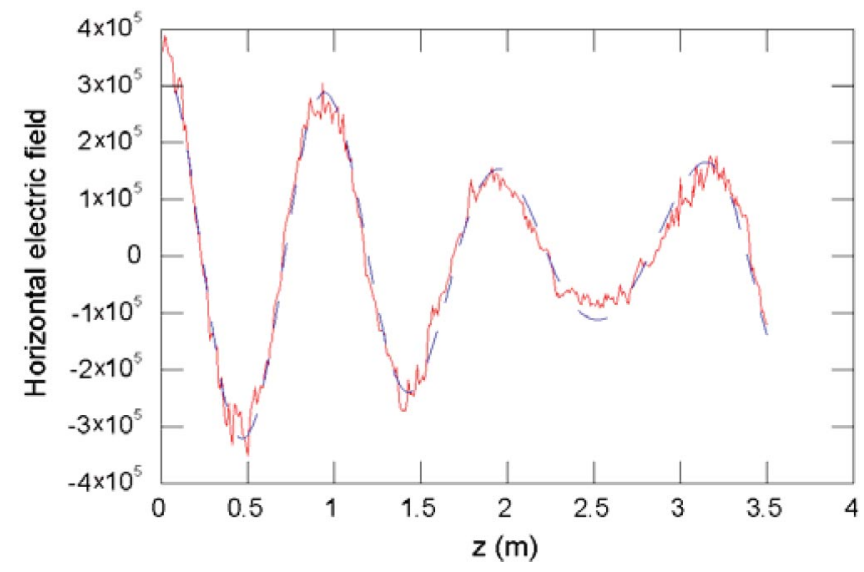

FIG. 14. (Color) Comparison of the electron centroid grid and analytic electric fields, using method 2 , without fractional ionization. The solid red line shows the electric field calculated on the simulation grid and the dashed blue line shows the electric field calculated by the analytic model. tually shortens the electron betatron period, and the betatron amplitude then attenuates, also in agreement with the simple model presented above.

\section{NOMINAL ACCELERATOR TRANSPORT CASES}

In this section, we will use the hybrid-fluid model to simulate some nominal long-pulse beam transport cases. In particular, we will model the ion-hose instability for various beam and transport parameters, for 5-meter long transport channels. The goal of these simulations is to identify key features of the instability, especially growth rates and saturation mechanisms. These insights will in turn be used to help interpret long-pulse simulations of the DARHT second-axis beam line, discussed in the following section.

For these cases, we will use a $4 \mathrm{kA}$ beam at $20 \mathrm{MeV}$. We will assume that the gas pressure is $5 \times\left(10^{-7}\right)$ torr and the cross section for releasing hydrogen ions is $4 \times$ $\left(10^{-23}\right) \mathrm{m}^{2}$. Hydrogen is used for these simulations because its light weight will lead to the shortest ion oscillation period and greatest growth rate.

The ion-hose instability has a complicated functional dependency on axial position and time. It is felt that plots of both ion and electron centroid motion at various axial locations as a function of time are the most illustrative of the physical mechanism, and will be the primary tool for this discussion and the one in the following section.

We will consider four $20 \mathrm{MeV}$ cases, with initial primary beam sinusoidal offset amplitudes of 0.1 to $1 \mathrm{~mm}$. First, we consider the case where the beam is matched in a uniform magnetic field with an edge radius of $3 \mathrm{~mm}$. The ion oscillation period for this case is $4 \mathrm{nsec}$. We will drive this instability with a sinusoidal electron-beam centroid offset (with a maximum of $0.1 \mathrm{~mm}$ ) also with a period of $4 \mathrm{nsec}$. Following that, we will observe the ion-hose stability for the case where the beam is focused to about $3 \mathrm{~mm}$ edge radius with periodic thin solenoids. Next, we consider the same beam, but with a $3 \mathrm{~cm}$ edge radius, and with an initial centroid offset period of $40 \mathrm{nsec}$. The final simulation will be the same as the $3 \mathrm{~mm}$ case with solenoidal focusing, but with an electron-beam centroid offset period of $8 \mathrm{nsec}$ to observe nonresonant excitation.

\section{A. 20-MeV electron-beam, 3-mm edge radius}

In this case, we will consider a $20-\mathrm{MeV}, 4-\mathrm{kA}$ beam, with a 3-mm radius, with a maximum offset amplitude of $0.1 \mathrm{~mm}$ with a resonant period of $4 \mathrm{nsec}$. The beam is matched in a solenoidal field of $0.125 \mathrm{~T}$. With these parameters, the scaling factor $I /\left(a^{2} B_{0}^{2}\right)$ is about 28 (so the ionization model used is very accurate). In Fig. 15 we see the evolution of the ion centroid as a function of time at different axial locations. The electron-beam centroid evolution is shown in Fig. 16. Each electron slice in these simulations corresponds to $0.4 \mathrm{nsec}$, for a total pulse length of $200 \mathrm{nsec}$. For these parameters, the ion oscillation 

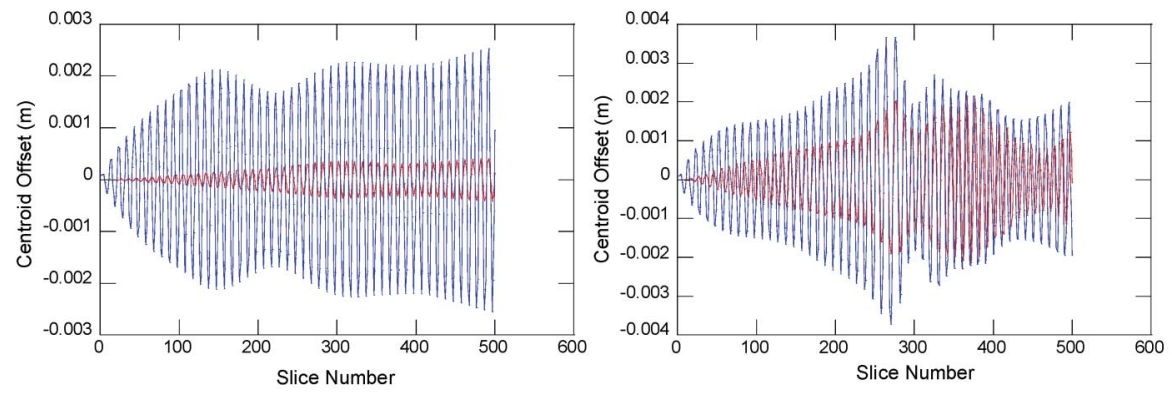

(a)

(b)
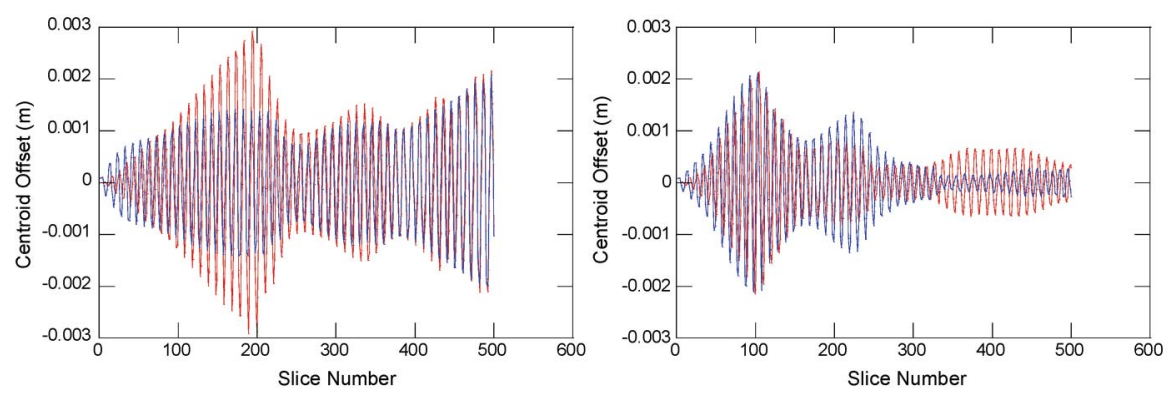

(c)

(d)

FIG. 15. (Color) Ion centroid evolution for 20-MeV, 3-mm, resonant beam case at different axial positions. Each slice is $0.4 \mathrm{nsec}$ long. The red line is for the horizontal offset and the blue line is for the vertical offset. (a) $z=0.625 \mathrm{~m}$, (b) $z=1.25 \mathrm{~m}$, and (c) $z=2.5 \mathrm{~m}$, and (d) $z=5 \mathrm{~m}$.

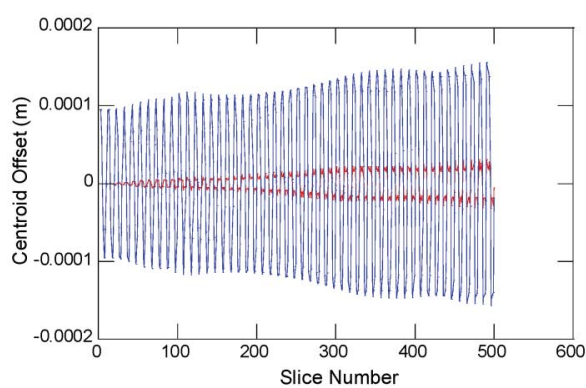

(a)

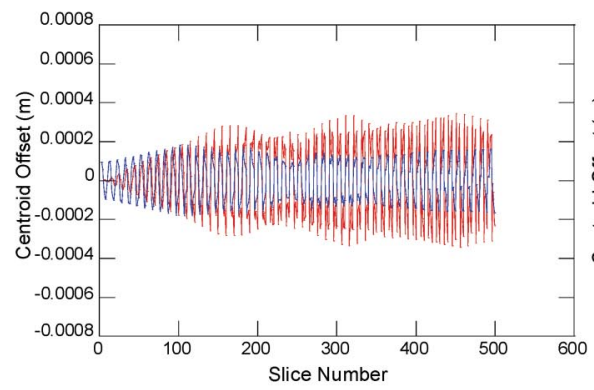

(c)

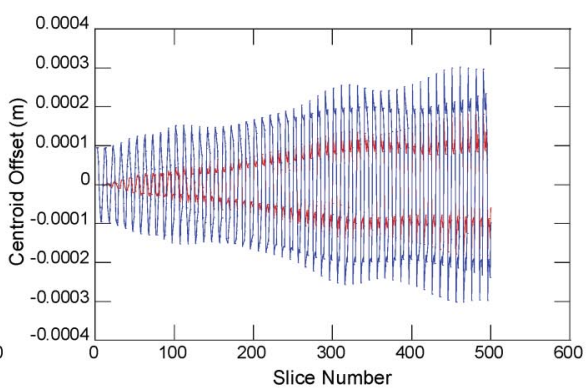

(b)

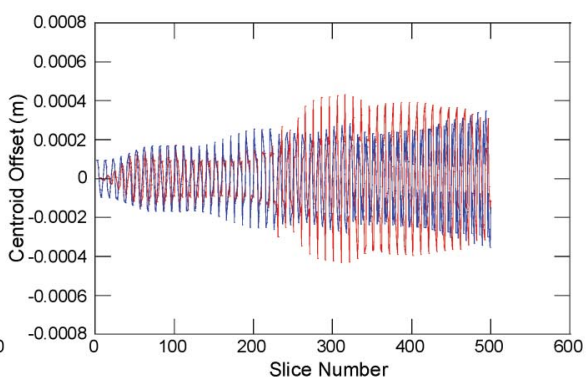

(d)

FIG. 16. (Color) Electron-beam centroid evolution for $20-\mathrm{MeV}, 3-\mathrm{mm}$, resonant beam case at different axial positions. Each slice is $0.4 \mathrm{nsec}$ long. The red line is for the horizontal offset and the blue line is for the vertical offset. (a) $z=0.625 \mathrm{~m}$, (b) $z=1.25 \mathrm{~m}$, and (c) $z=2.5 \mathrm{~m}$, and (d) $z=5 \mathrm{~m}$. 
period is about $4 \mathrm{nsec}$. The ion centroid amplitude reaches a couple of millimeters, but the axial magnetic field restricts the electron-beam centroid offset to about $0.2 \mathrm{~mm}$. The ion centroid oscillations saturate once the ions move out of the potential well of the electron beam. The electron centroid growth does not saturate for this pulse length at $0.625 \mathrm{~m}$, is close to saturating at $1.25 \mathrm{~m}$, and saturates at $2.5 \mathrm{~m}$ and beyond. This saturation is also reflected in the ion centroid evolutions. At 2.5 and $5 \mathrm{~m}$, the ion centroid evolution shows that the ions lose coherence with the electrons, causing the saturation. The electron centroid amplitude is suppressed by the confining axial magnetic field to about one tenth that of the ions.

\section{B. 20-MeV electron beam, 3-mm edge radius with periodic focusing}

In this case, we will consider a $20-\mathrm{MeV}$, 4-kA beam, with a 3-mm radius, also with a maximum centroid offset of $0.1 \mathrm{~mm}$. The beam is matched with a periodic lattice of $10-\mathrm{cm}$ long solenoids, spaced $1 \mathrm{~m}$ apart. Here, the scaling factor $I /\left(a^{2} B_{0}^{2}\right)$ is about 3 in the solenoids and infinite between them. Since the solenoids constitute a small portion of the transport, we anticipate the simulation model will be quite accurate. The rms beam radius evolution over the transport distance is shown in Fig. 17. As in the previous case, $0.4 \mathrm{nsec}$ slices are used. However, the instability did not saturate in $200 \mathrm{nsec}$, so a total of 2500 slices was used, for a total electron pulse length of $1 \mu \mathrm{sec}$. In Fig. 18 we see the evolution of the ion centroid as a function of time at different axial locations. The electronbeam centroid evolution is shown in Fig. 19. The plots of the ion centroid and electron centroid evolutions are at the locations of the periodic solenoids. We see that the ion centroids have a similar amplitude to the previous case with uniform focusing (about $2 \mathrm{~mm}$ ), and now the electron centroids have comparable amplitudes, a factor of 5 greater than the previous case. A major difference introduced in

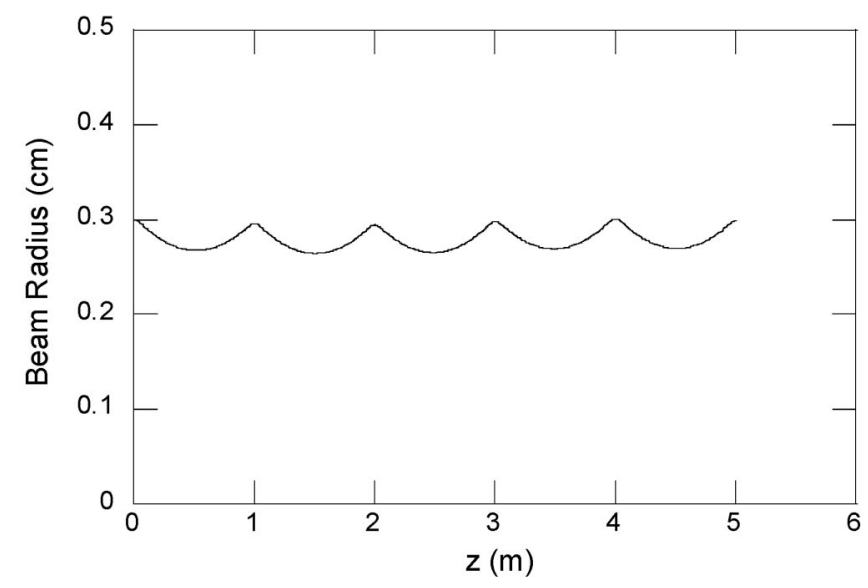

FIG. 17. Beam edge radius versus transport distance, for the period focusing case. $10-\mathrm{cm}$ long solenoids are located at meter intervals, with strengths of $0.41 \mathrm{~T}$. this case relative to the uniform focusing cases is that here the electron and ion centroids will hunt for a resonance, which is clearly seen in the electron centroid plots at 3, 4, and $5 \mathrm{~m}$ where a new period is established and in the ion centroid plots where the ion centroids lose coherence. We see that at axial distances of $3 \mathrm{~m}$ and beyond, the period of the ion and electron centroid oscillations change dramatically. This shift is seen clearly in Fig. 19(f).

\section{20-MeV electron beam, 3-cm edge radius}

In this case, we will consider a $20 \mathrm{MeV}, 4 \mathrm{kA}$ beam, with a $3 \mathrm{~cm}$ radius. A very low axial magnetic field is used to match the rms beam radius, leading to a very large scaling factor $I /\left(a^{2} B_{0}^{2}\right)$. For this beam radius, the ion period is $40 \mathrm{nsec}$, and in this simulation the electron centroid offset period is also $40 \mathrm{nsec}$ to resonantly excite the instability (with an initial maximum amplitude of $1 \mathrm{~mm}$ ). In Fig. 20 we see the evolution of the ion centroid as a function of time at different axial locations. The electron-beam centroid evolution is shown in Fig. 21. Ion amplitude saturation again occurs for radii above $2 \mathrm{~cm}$, and the electron amplitude does not show saturation either over the $5 \mathrm{~m}$ or over the $2 \mu \mathrm{sec}$ pulse length. The total amplitude of the instability is about the same as the case with a $3-\mathrm{mm}$ radius, but the relative effect is smaller, because of the larger beam size.

\section{20-MeV electron beam, 3-mm edge radius, nonresonant oscillations}

In this case, we will consider a $20-\mathrm{MeV}, 4-\mathrm{kA}$ beam, with a $3-\mathrm{mm}$ radius. The beam is matched in a solenoidal field of $0.125 \mathrm{~T}$. The difference between this case and the first case is that the initial beam centroid offset has a period of $8 \mathrm{nsec}$, which is not resonant with the ion oscillation period of $4 \mathrm{nsec}$. Ths slices in this case are each $0.8 \mathrm{nsec}$ long, with a total simulation pulse length of $400 \mathrm{nsec}$. In Fig. 22 we see the evolution of the ion centroid as a function of time at different axial locations. The electronbeam centroid evolution is shown in Fig. 23. The saturated amplitudes are similar to those seen for the resonantly driven case (about $0.2 \mathrm{~mm}$ ), and by $1.25 \mathrm{~m}$, the ion centroid oscillation has a dominant period of $4 \mathrm{nsec}$. In addition, the electron centroids show a dominant oscillation of $4 \mathrm{nsec}$ by $5 \mathrm{~m}$. It appears that resonant excitation is not needed, although nonresonant excitation will delay saturation both in terms of axial location and time within the pulse itself.

\section{E. Discussion}

The results of the previous cases can be summarized by the following statements. Application of a uniform external magnetic field will suppress the amplitude of the electron centroid oscillations, as described in [3]. The ion oscillations are limited by the size of the electron-beam potential 

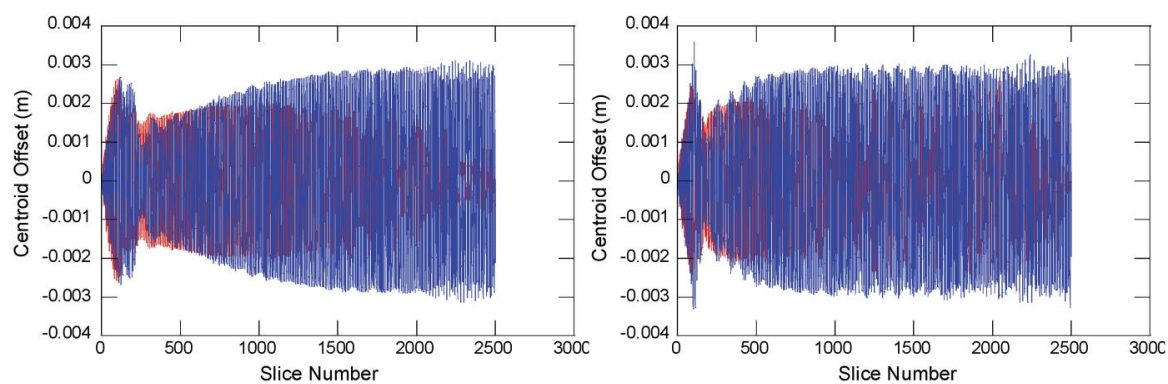

(a)

(b)
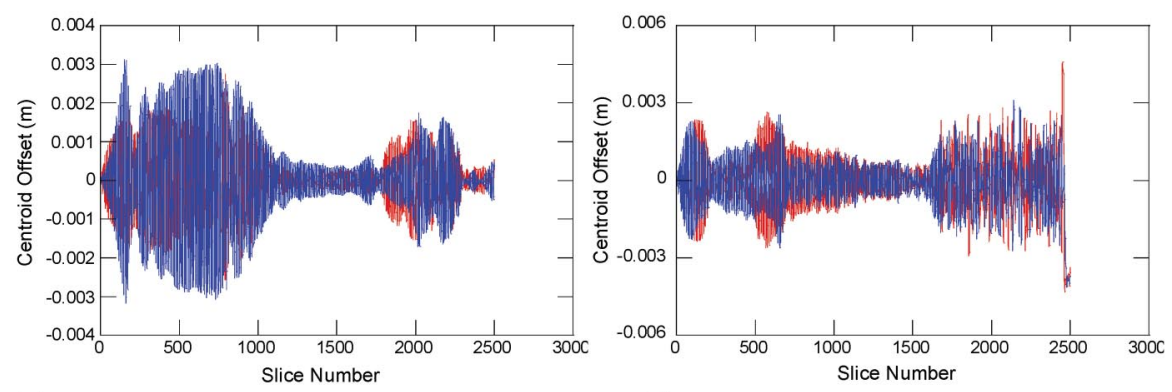

(c)

(d)

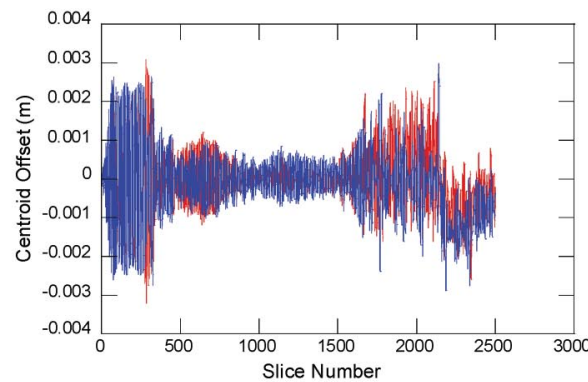

(e)

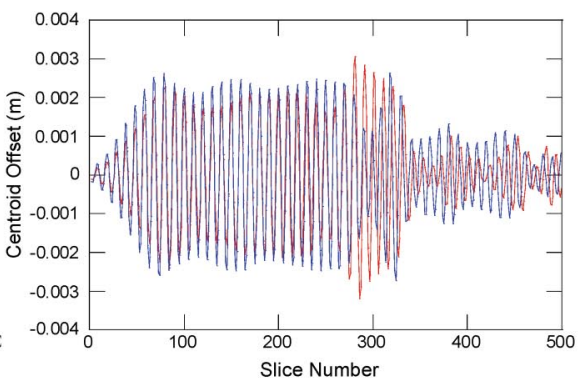

(f)

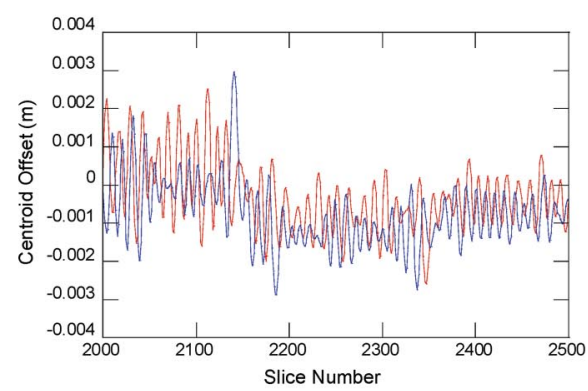

(g)

FIG. 18. (Color) Ion centroid evolution for $20-\mathrm{MeV}, 3-\mathrm{mm}$, periodic-focusing beam case at different axial positions. Each slice is $0.4 \mathrm{nsec}$ long. The red line is for the horizontal offset and the blue line is for the vertical offset. (a) $z=1 \mathrm{~m}$, (b) $z=2 \mathrm{~m}$, (c) $z=3 \mathrm{~m}$, (d) $z=4 \mathrm{~m}$, (e) $z=5 \mathrm{~m}$, (f) blowup of first 500 slices at $z=5 \mathrm{~m}$, and (g) blowup of last 500 slices at $z=5 \mathrm{~m}$.

well. Nonresonant excitation shows roughly equivalent results to resonant excitation. Periodic focusing reduces the suppression of the electron centroids and lead to greater saturated amplitudes, although the initial growth rate is about the same. The ion and electron centroids are allowed to hunt for resonances with periodic focusing. Larger beam sizes will decrease the relative effect of the instability.

\section{DARHT BEAM LINE ION-HOSE SIMULATIONS}

The DARHT second-axis accelerator is a long-pulse accelerator, designed for a $2-\mu \mathrm{sec}$ electron pulse. The accelerator has more-or-less continuous focusing along it, with long sections with reduced or no field only in the early part and in the downstream transport 


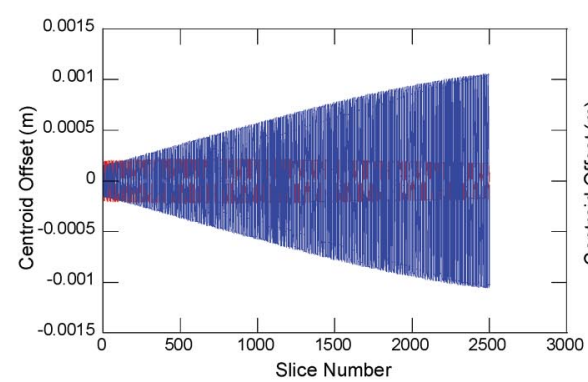

(a)

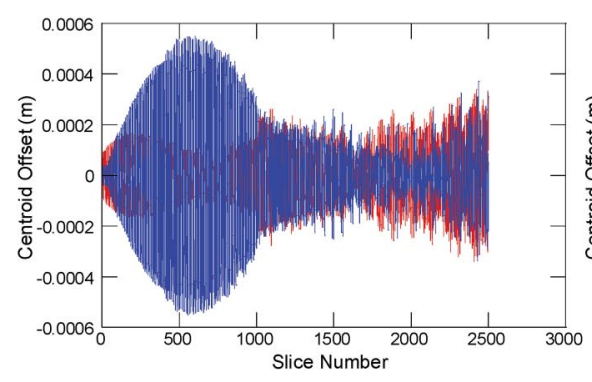

(c)

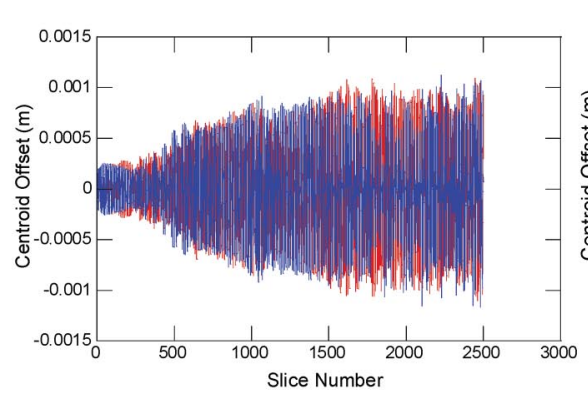

(e)

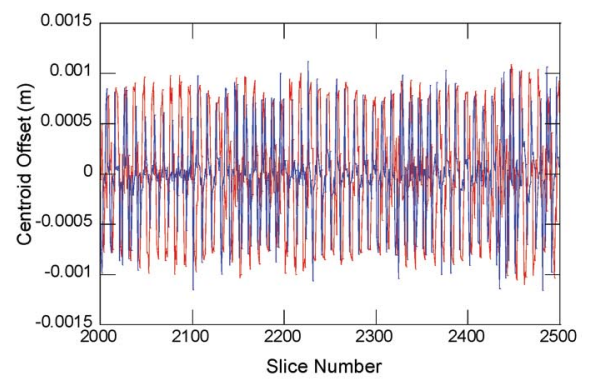

(g)

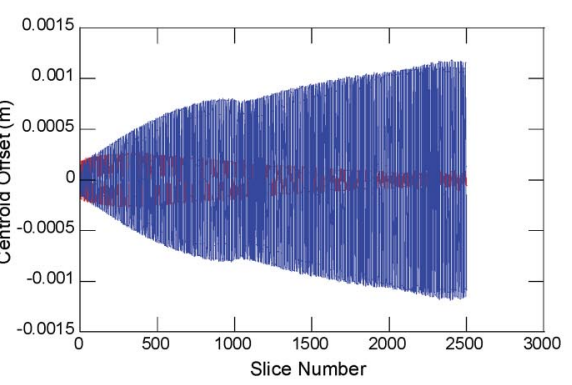

(b)

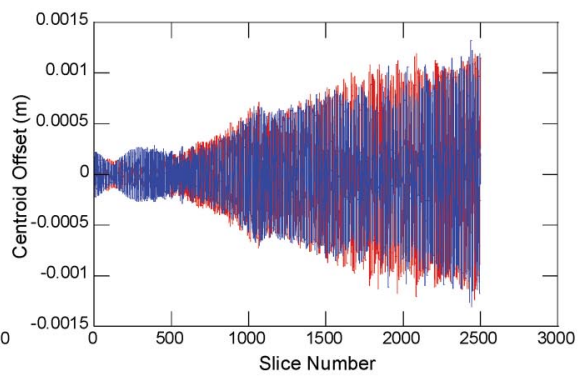

(d)

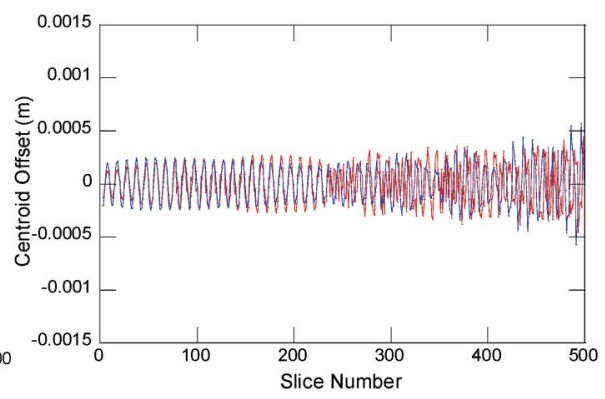

(f)

FIG. 19. (Color) Electron-beam centroid evolution for 20-MeV, 3-mm, periodic-focusing beam case at different axial positions. Each slice is $0.4 \mathrm{nsec}$ long. The red line is for the horizontal offset and the blue line is for the vertical offset. (a) $z=1 \mathrm{~m}$, (b) $z=2 \mathrm{~m}$, (c) $z=3 \mathrm{~m}$, (d) $z=4 \mathrm{~m}$, (e) $z=5 \mathrm{~m}$, (f) blowup of first 500 slices at $z=5 \mathrm{~m}$, and (g) blowup of last 500 slices at $z=5 \mathrm{~m}$.

after the last accelerating cell. The downstream transport after the accelerator is intended to match the electron beam into a kicker section which transforms the beam into a series of 50 to $100 \mathrm{nsec}$ pulses. The accelerator is intended to eventually produce a $18.4-\mathrm{MeV}, 2-\mathrm{kA}$ electron beam for fast radiography (initially a $1.4-\mathrm{kA}$ beam will be produced). The accelerator layout is shown in Fig. 24. The ion-hose instability can be suppressed for this accelerator with proper tuning. In this section, we will study the instability in an upstream section with discrete magnetic focusing and for an alternative tuning in the downstream transport where it can become significant 


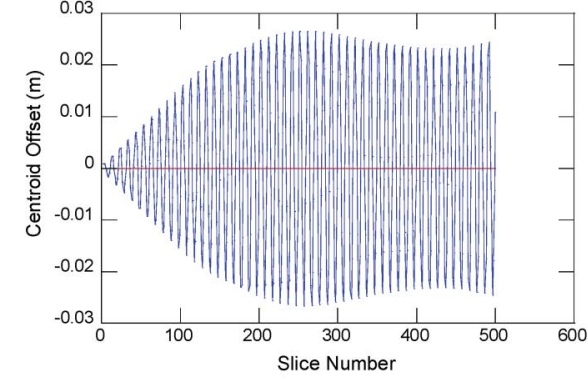

(a)

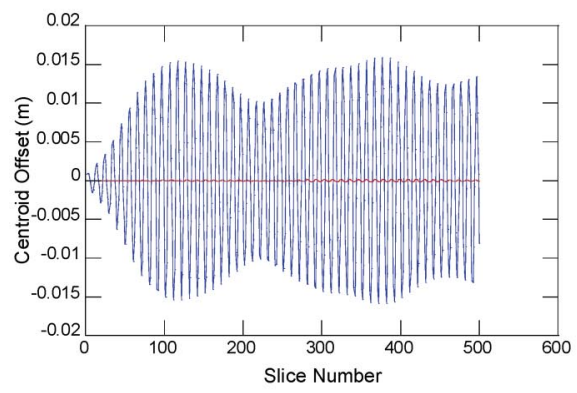

(c)

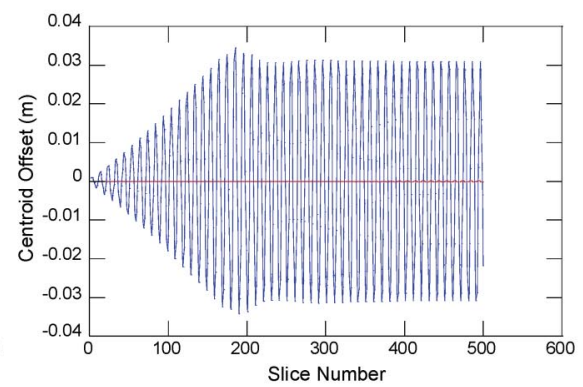

(b)

FIG. 20. (Color) Ion centroid evolution for $20-\mathrm{MeV}, 3-\mathrm{cm}$ beam case at different axial positions. Each slice is $4 \mathrm{nsec}$ long. The red line is for the horizontal offset and the blue line is for the vertical offset. (a) $z=1.25 \mathrm{~m}$, (b) $z=2 \mathrm{~m}$, and (c) $z=5 \mathrm{~m}$.

(basically transporting a smaller electron-beam radius). We will use a gas pressure in the beam pipe of $2 \times\left(10^{-7}\right)$ torr and cross section for water ions of $2 \times\left(10^{-22}\right) \mathrm{m}^{2}$. Water ions $\left(\mathrm{H}_{2} \mathrm{O}^{+}\right)$are considered the most likely candidate for ionization from impact ionization by the primary drive beam. A full analysis of the instability for the DARHT accelerator is beyond the scope of this paper. However, these examples can be used with the cases in the previous

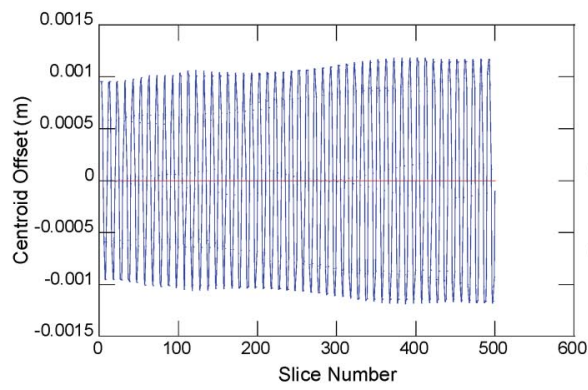

(a)

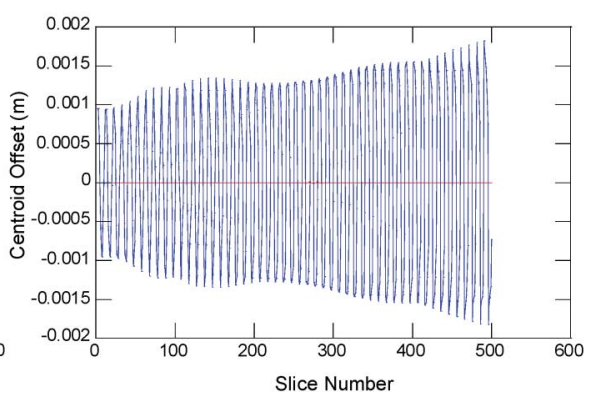

(b)

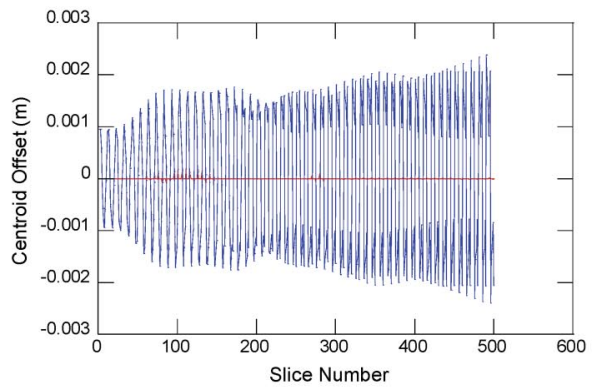

(c)

FIG. 21. (Color) Electron-beam centroid evolution for $20-\mathrm{MeV}, 3-\mathrm{cm}$ beam case at different axial positions. Each slice is 4 nsec long. The red line is for the horizontal offset and the blue line is for the vertical offset. (a) $z=1.25 \mathrm{~m}$, (b) $z=2.5 \mathrm{~m}$, and (c) $z=5 \mathrm{~m}$. 

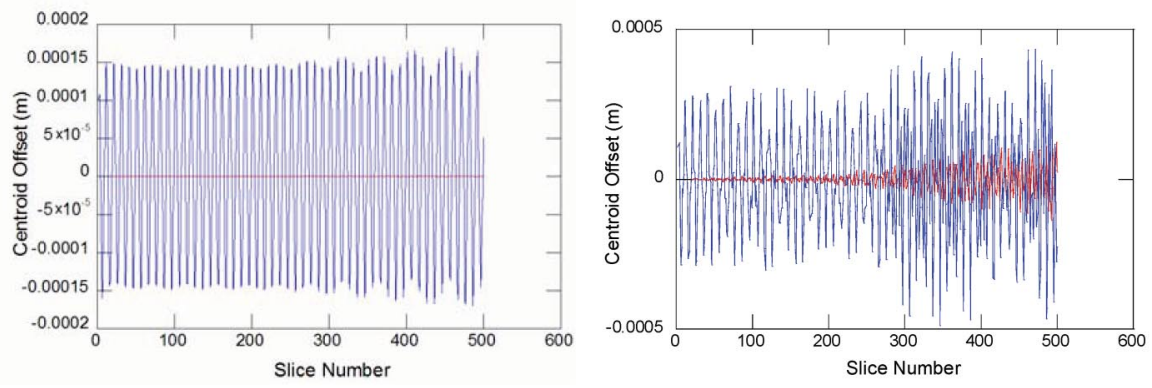

(a)

(b)

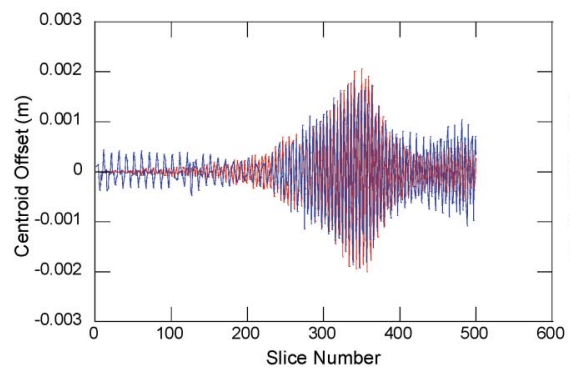

(c)

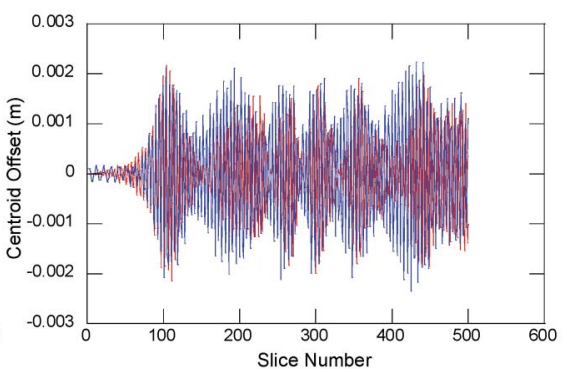

(d)

FIG. 22. (Color) Ion centroid evolution for $20-\mathrm{MeV}, 3-\mathrm{mm}$, nonresonant beam case at different axial positions. Each slice is $0.8 \mathrm{nsec}$ long. The red line is for the horizontal offset and the blue line is for the vertical offset. (a) $z=0.2 \mathrm{~m}$, (b) $z=0.625 \mathrm{~m}$, and (c) $z=1.25 \mathrm{~m}$, and (d) $z=5 \mathrm{~m}$.

section to build insight into the instability mechanism. In particular, the widely spaced focusing will encourage the instability, and the varying beam size will provide a very wide resonance.

\section{A. Commissioning geometry}

For the early part of the DARHT accelerator, we use the nominal commissioning tune, with beam radius, applied magnetic field, and beam emittance evolution shown in

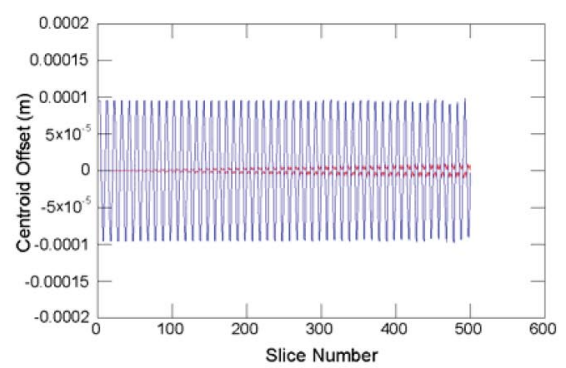

(b)

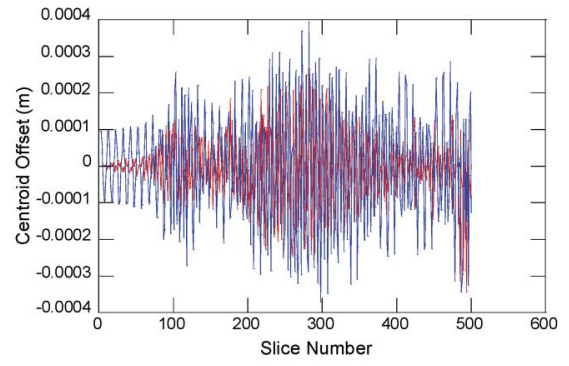

(d)

FIG. 23. (Color) Electron-beam centroid evolution for 20-MeV, 3-mm, nonresonant beam case at different axial positions. Each slice is $0.8 \mathrm{nsec}$ long. The red line is for the horizontal offset and the blue dashed line is for the vertical offset. (a) $z=0.2 \mathrm{~m}$, (b) $z=$ $0.625 \mathrm{~m}$, and (c) $z=1.25 \mathrm{~m}$, and (d) $z=5 \mathrm{~m}$. 


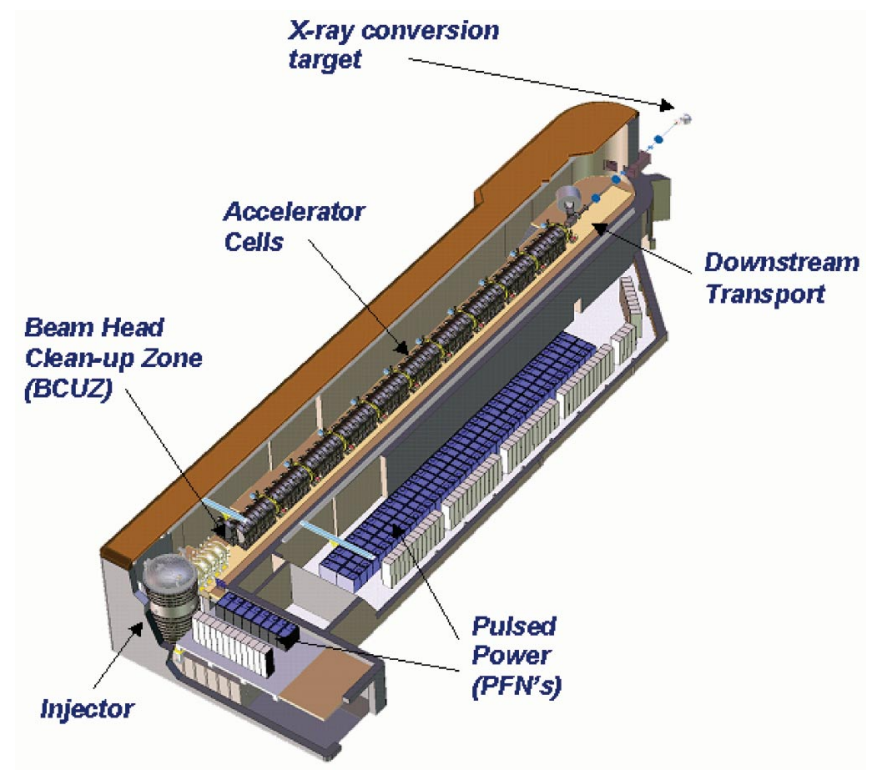

FIG. 24. (Color) Schematic of the DARHT second-axis accelerator, showing the layout of the injector, beam head clean-up zone, accelerator cells, and downstream transport.

Fig. 25. The commissioning geometry is slightly different than the upstream geometry for the final accelerator configuration, because additional solenoids are installed in the axial region from 9 to $12.5 \mathrm{~m}$ for better matching into the injector commissioning diagnostics. The beam energy grows from $2.6 \mathrm{MeV}$ to $4.0 \mathrm{MeV}$ in this section, with a beam current of $1410 \mathrm{~A}$. There are 8 accelerator cells, each with $175 \mathrm{kV}$, centered at axial positions of 2.1, 2.6, 3.1, 3.6, $4.2,4.7,5.2$, and $5.7 \mathrm{~m}$. The beam radius, injected from a diode, starts at about $7 \mathrm{~cm}$ and is focused to about $2 \mathrm{~cm}$. A region of the transport intended to scrape off the beam head, called the beam cleanup zone (BCUZ), is from about 6 to about $9 \mathrm{~m}$, followed by special solenoids used only for the injector commissioning. The BCUZ will also be part of the final accelerator configuration. For the commissioning tune, the solenoids in the BCUZ are detuned to provide smooth transport, instead of a series of waists. We see the magnetic field holes due to the discrete location of the solenoids in this section, in Fig. 25(b). The emittance evolution, shown in Fig. 25(c) is dominated by transverse plasma oscillations and other nonthermal effects, and is well understood [25]. For these parameters, the normalized scaling factor $I /\left(a^{2} B_{0}^{2}\right)$ is typically about 50 , and the assumption in numerical model neglecting the ionization electrons should be quite accurate.

The electron centroid evolution at the end of the commissioning geometry $(12.5 \mathrm{~m})$ is shown in Fig. 26, for various initial offset periods, ranging from about 100 to $600 \mathrm{nsec}$. For this analysis, an $8 \mu$ sec electron beam was used in the simulations, because the instability will not saturate in $2 \mu \mathrm{sec}$, for any of the initial offset periods. For all cases, the maximum initial centroid offset was $0.1 \mathrm{~mm}$, and 1000 electron slices of $8 \mathrm{nsec}$ apiece were used. We see a relatively narrow resonance, peaked at a drive frequency of about $2.3 \mathrm{MHz}$ (440 nsec period), with a bandwidth of

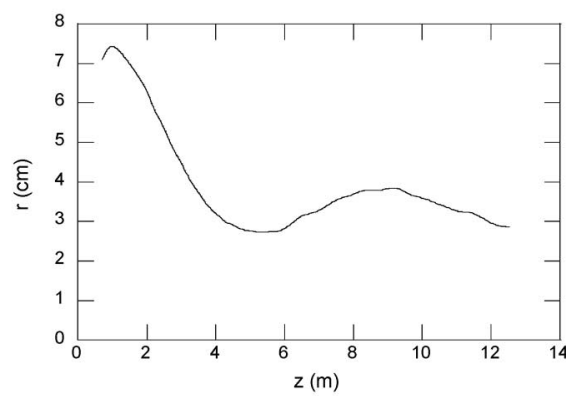

(a)

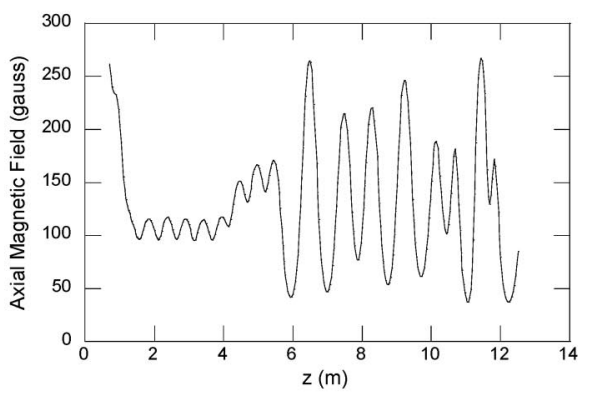

(b)

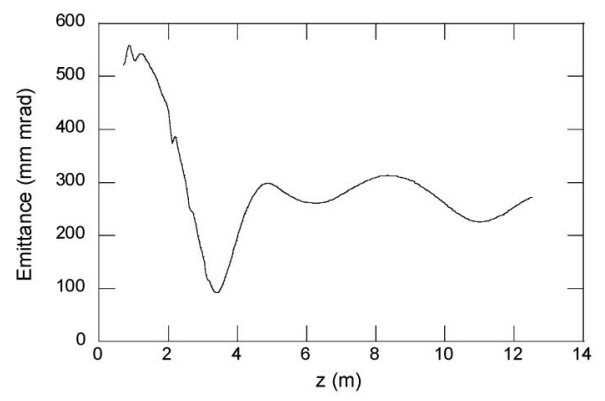

(c)

FIG. 25. Commissioning tune: (a) rms beam radius evolution, (b) applied external magnetic field, and (c) rms beam emittance evolution. 

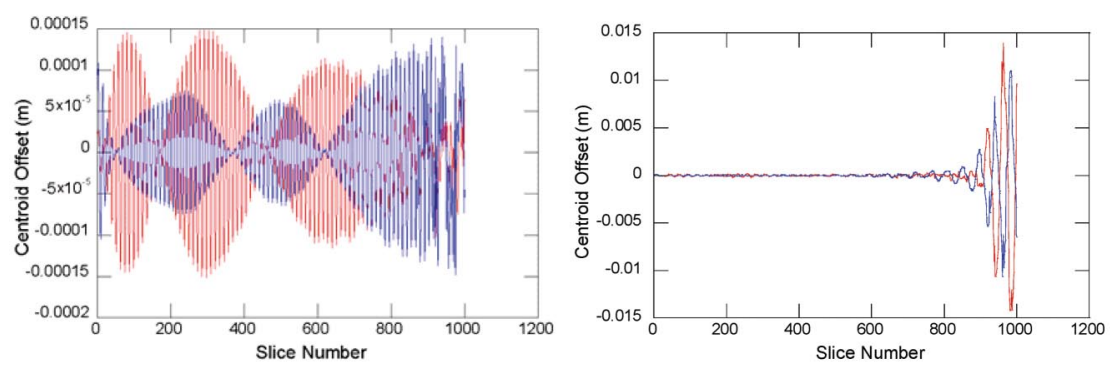

(a)

(b)
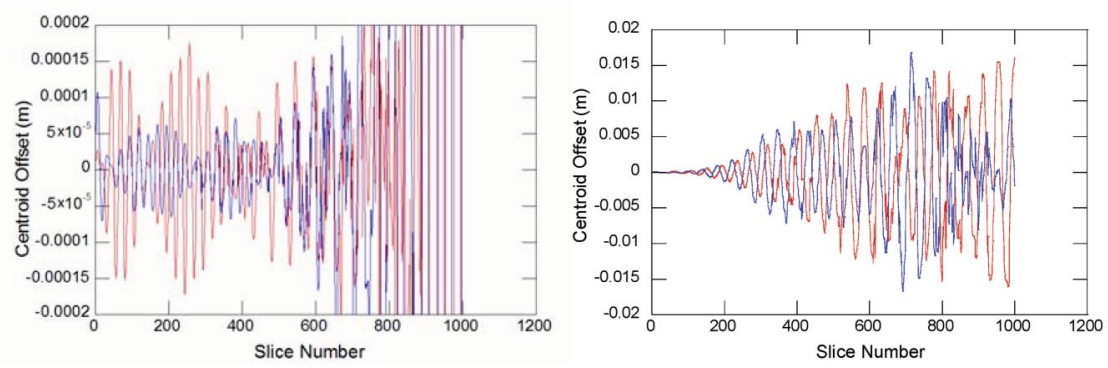

(c)

(d)

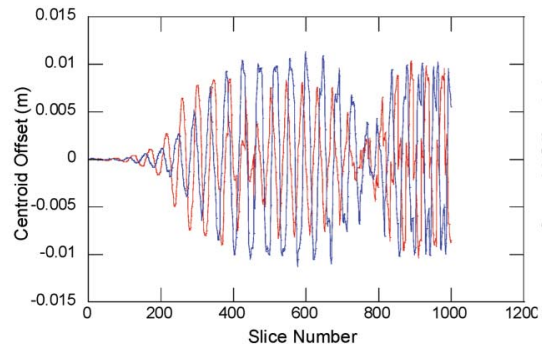

(e)

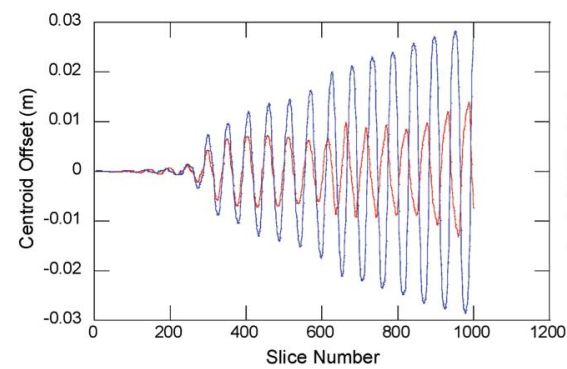

(g)

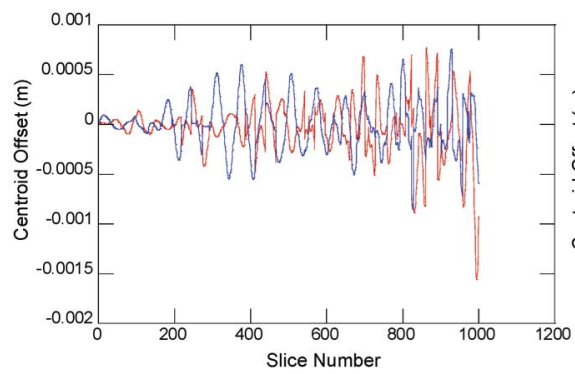

(i)

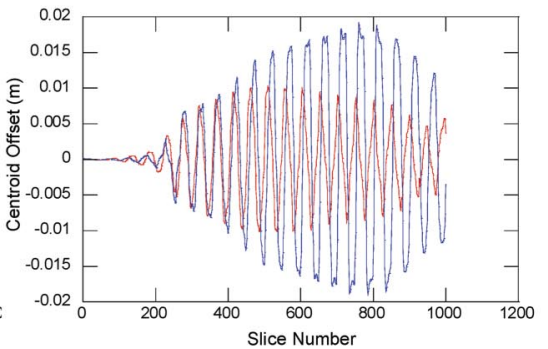

(f)

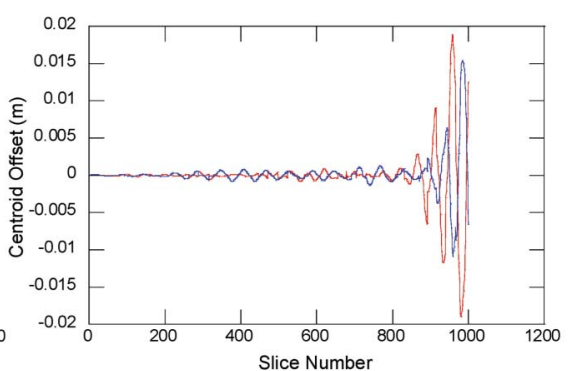

(h)

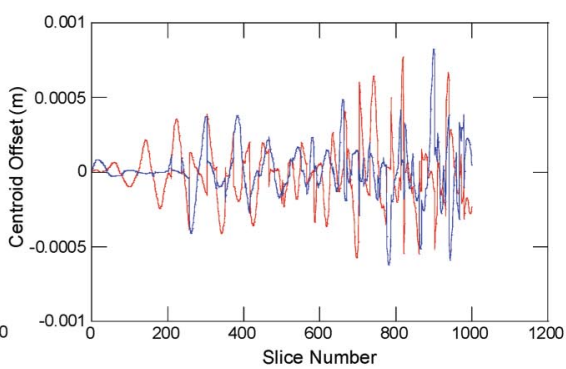

(j)

FIG. 26. (Color) Electron centroids at $12.5 \mathrm{~m}$ for an $8 \mu \mathrm{sec}$ beam ( $2 \mu \mathrm{sec}$ has elapsed by the 250th slice). The red line is for the horizontal offset and the blue line is for the vertical offset. (a) period of $96 \mathrm{nsec}$, (b) period of $200 \mathrm{nsec}$, (c) period of $200 \mathrm{nsec}$ on different vertical scale, showing same envelope as the $96 \mathrm{nsec}$ case, (d) period of $304 \mathrm{nsec}$, (e) period of $352 \mathrm{nsec}$, (f) period of $400 \mathrm{nsec}$, (g) period of $440 \mathrm{nsec}$, (h) period of $480 \mathrm{nsec}$, (i) period of $520 \mathrm{nsec}$, and (j) period of $600 \mathrm{nsec}$. 


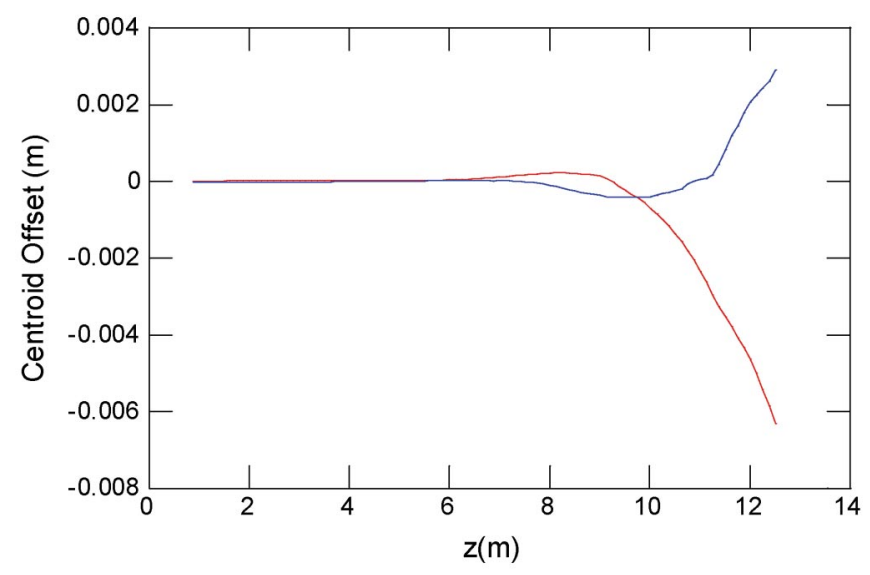

FIG. 27. (Color) Centroid of slice 800 (6.4 $\mu$ sec into pulse) versus distance for the commissioning geometry. The red line is for the horizontal centroid and the blue line is for the vertical centroid.

about $0.8 \mathrm{MHz}$. At resonance [Fig. 26(g)], we see clean, single frequency, growth of the ion-hose instability, with no frequency hopping. Off resonance, we see beating and frequency hopping. Note that the resonance is very onesided, and the response quickly drops off as the driving frequency is decreased. In Fig. 26(a), we see the signature of driving the ion-hose instability far off resonance. No amplitude growth is seen. At a period of $200 \mathrm{nsec}$ [Figs. 26(b) and 26(c)], the same low amplitude signature is seen, until late in the pulse when frequency hopping is made to approximately the second subharmonic and reso- nance is found. At resonance [Fig. 26(g)], the growth of the electron-beam centroid oscillations reaches $3 \mathrm{~cm}$ after $8 \mu \mathrm{sec}$. The maximum growth after $2 \mu \mathrm{sec}$ (the DARHT pulse length) is less than $5 \mathrm{~mm}$.

In Fig. 27, we plot the axial evolution of the centroid of the 800th electron slice, to verify that the majority of the exponential growth does in fact happen in the region of the periodic solenoids.

In Figs. 28 and 29, we plot the ion and electron evolutions for an excitation frequency of $2.3 \mathrm{MHz}$, at axial distances of $5,7.5,8.75$, and $10 \mathrm{~m}$. The drive frequency is off resonance at the local condition at $5 \mathrm{~m}$, and very little ion oscillations are seen, and no growth in the electron amplitudes are seen. At $7.5 \mathrm{~m}$, some limited growth is seen, but both the ion and electron centroids appear to saturate at a low level. Both centroids start seeing exponential growth at $8.75 \mathrm{~m}$, and have large growth rates at $10 \mathrm{~m}$. A key insight into the mechanism is that by $10 \mathrm{~m}$, the amplitude of the electron centroids is comparable to that of the ion centroids, suggesting that the mechanism damping effect from the confinement of the electrons from the external magnetic field is failing for the periodic field structure. The drive frequency stays in resonance over the range from 5 to $10 \mathrm{~m}$ because there is very little change in the beam radius.

\section{B. Downstream transport}

After the last DARHT accelerating cell is a region called the downstream transport. We will use a modified focusing tune where the $19-\mathrm{MeV}, 1.41-\mathrm{kA}$ electron beam is about $2 \mathrm{~mm}$ at the end of the accelerator section just preceding

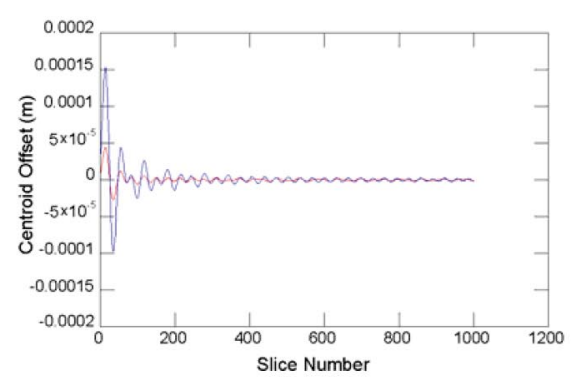

(a)

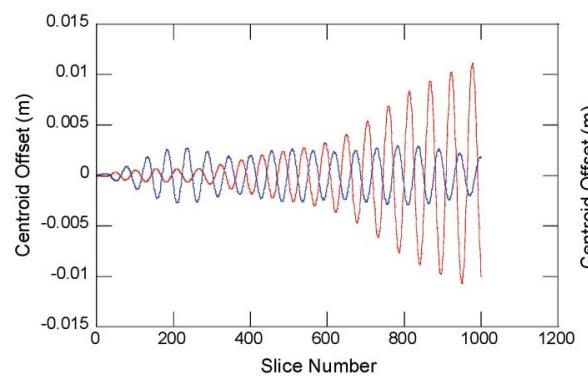

(c)

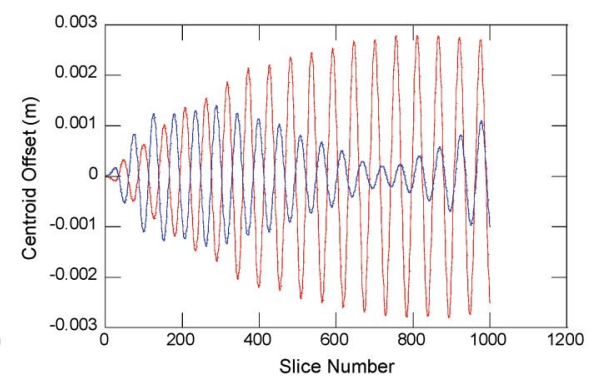

(b)

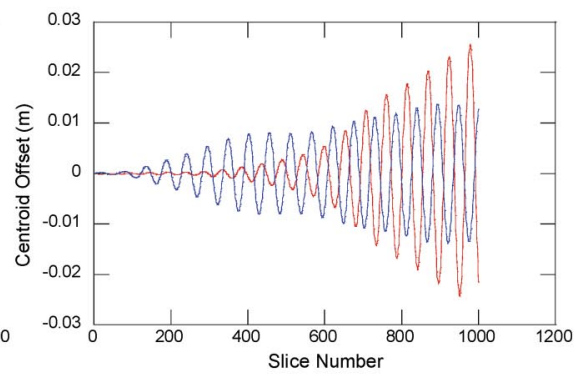

(d)

FIG. 28. (Color) Ion centroids at (a) $5 \mathrm{~m}$, (b) $7.5 \mathrm{~m}$, (c) $8.75 \mathrm{~m}$, and (d) $10 \mathrm{~m}$, for an $8 \mu$ sec beam in the commissioning geometry, for an initial offset period of $440 \mathrm{nsec}$. The red line is for the horizontal offset and the blue line is for the vertical offset. 


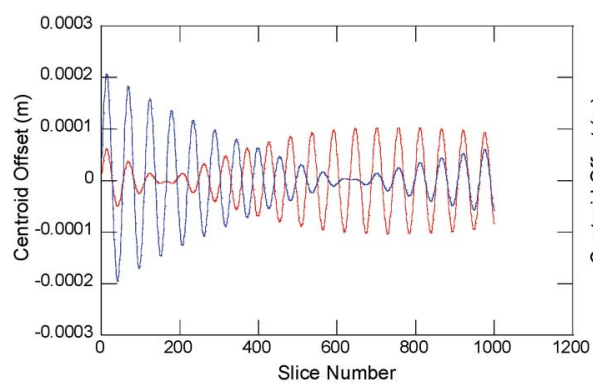

(a)

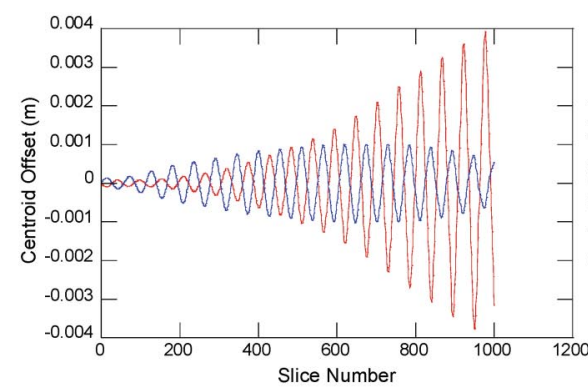

(c)

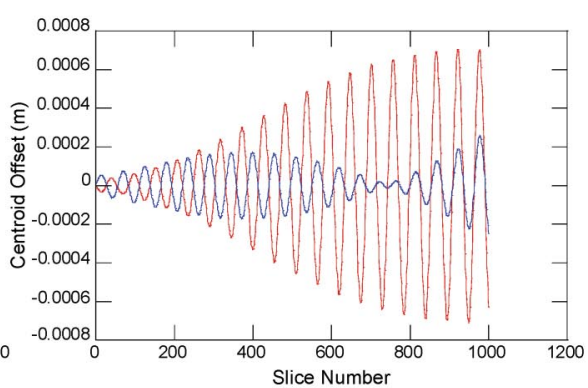

(b)

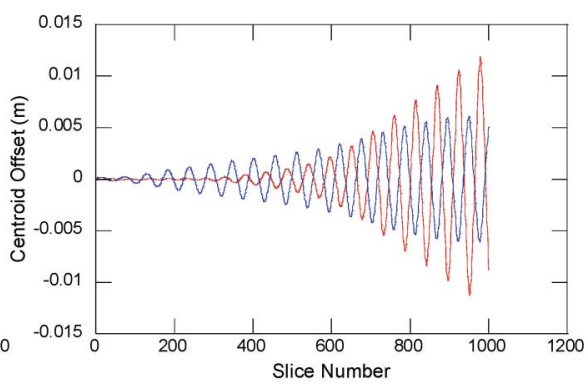

(d)

FIG. 29. (Color) Electron centroids at (a) $5 \mathrm{~m}$, (b) $7.5 \mathrm{~m}$, (c) $8.75 \mathrm{~m}$, and (d) $10 \mathrm{~m}$, for an $8 \mathrm{msec}$ beam in the commissioning geometry, for an initial offset period of $440 \mathrm{nsec}$. The red line is for the horizontal offset and the blue line is for the vertical offset.

this section, and evolves to about $5.3 \mathrm{~mm}$ at the end of this section. Focusing solenoids are located at about 1.8 and $7.6 \mathrm{~m}$. The beam rms radius, external focusing magnetic field, and beam emittance are shown in Fig. 30. For this simulation, hard-edged solenoidal fields were assumed. The normalized scaling factor $I /\left(a^{2} B_{0}^{2}\right)$ is about 2 in the solenoids but infinite between them. Since the solenoid lengths are short and we know the growth of the instability

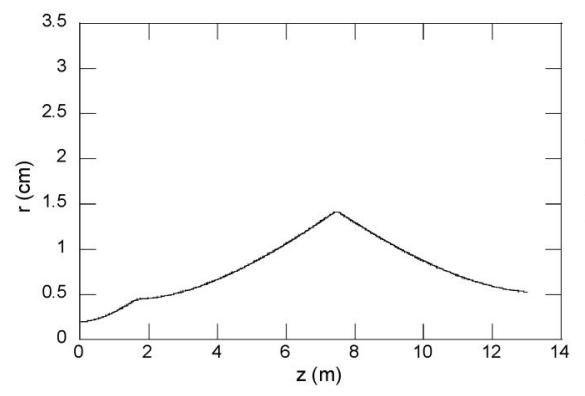

(a)

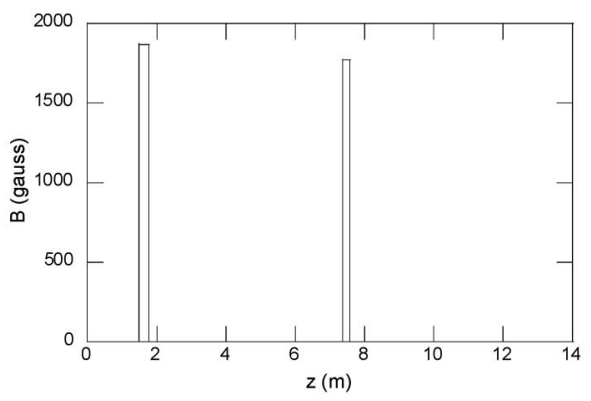

(b)

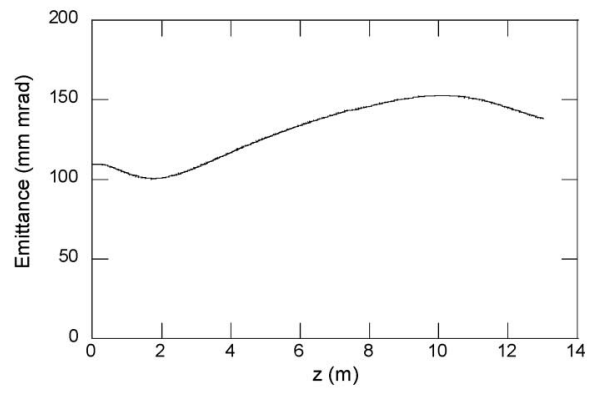

(c)

FIG. 30. Downstream tune: (a) rms beam radius evolution, (b) applied external magnetic field, and (c) rms beam emittance evolution. 


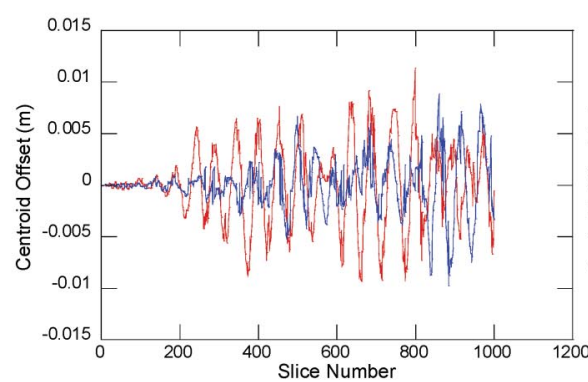

(a)

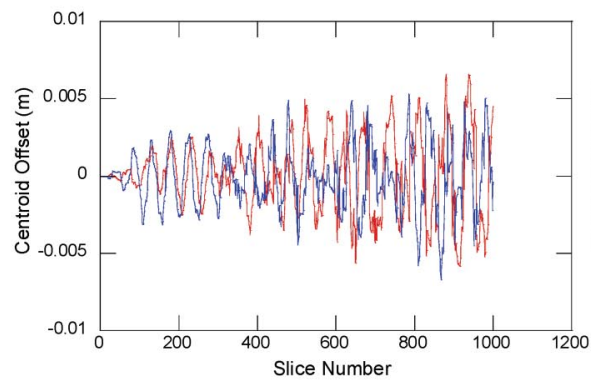

(c)

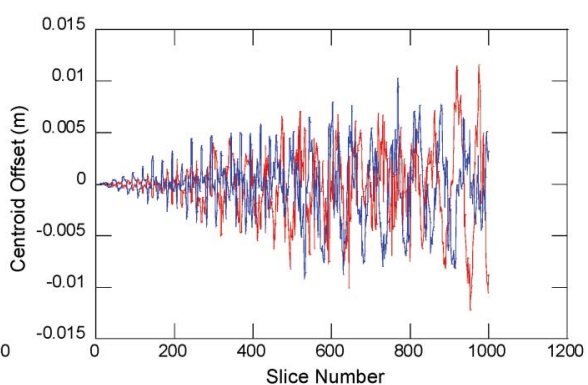

(b)

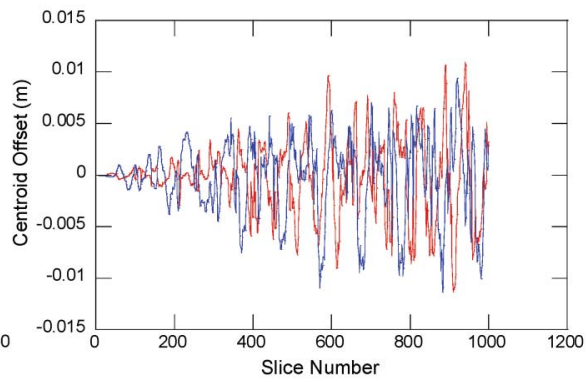

(d)

FIG. 31. (Color) Electron centroids at $13 \mathrm{~m}$ (a) $26 \mathrm{nsec}$ period, (b) $50 \mathrm{nsec}$ period, (c) $100 \mathrm{nsec}$ period, and (d) $200 \mathrm{nsec}$ period, for a $2 \mathrm{msec}$ beam in the downstream transport. The red line is for the horizontal offset and the blue line is for the vertical offset.

is suppressed by high axial fields anyway, the error in the solenoids is not significant.

For this case, the smaller beam radius leads to a much shorter ion oscillation period, and the simulations are for a total electron beam pulse length of $2 \mu \mathrm{sec}$, using 1000 slices of 2 nsec apiece. In Fig. 31, we plot the electron centroid offset at $13 \mathrm{~m}$ (the end of this transport section), for the case of an initial offset maximum of $0.1 \mathrm{~mm}$, for offset periods ranging from 26 to $200 \mathrm{nsec}$. In direct contrast to the previous case (the commissioning geometry), we now see an extremely wide resonance, essentially equally driven at all frequencies. The resonance is dominated by an oscillation with a $80 \mathrm{nsec}$ period, for all cases. Note that the large change in beam radius (from 2 to $15 \mathrm{~mm}$ at its maximum) allows a large range of drive frequencies to couple into the instability. In Fig. 32, we plot the axial evolution of the electron centroid of the 800th slice, at $1.6 \mu \mathrm{sec}$ into the electron pulse. The centroid definitely increases after the second solenoid, at about $7.6 \mathrm{~m}$. This large magnification of the centroid offset is caused by the large zero-current phase advance of the solenoid. The magnification of the centroid offset from periodic focusing is a form of the common thin-lens instability which occurs if the focal length of the lenses is less than one quarter of the lens separation.

In Fig. 33, we plot the ion centroid evolution at 2.5, 5, 7.5 , and $10 \mathrm{~m}$, for an initial offset period of $200 \mathrm{nsec}$. In Fig. 34, we plot the electron centroid evolution at the same locations. The ion centroid contains a strong $50 \mathrm{nsec}$ com- ponent by $2.5 \mathrm{~m}$. This oscillation period increases to about $100 \mathrm{nsec}$ by $7.5 \mathrm{~m}$, indicating the that oscillations are staying in phase with the local resonance of the ions due to the local beam size. The electron centroid oscillations contain all previous frequency components, and are rich in various components after $2.5 \mathrm{~m}$. As shown in the periodic case in Sec. IV, the centroids are allowed to find, and drive, the local resonances. The second solenoid acts as a lens magnifying the magnitude of these oscillations.

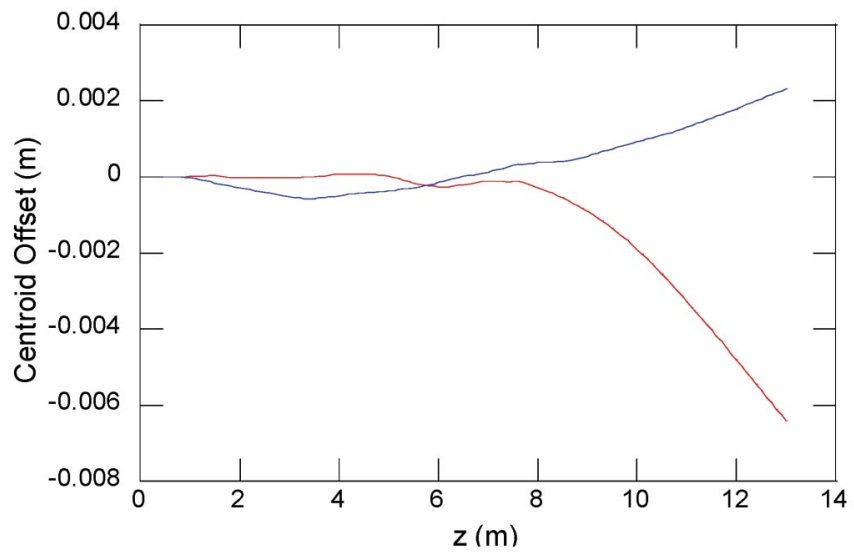

FIG. 32. (Color) Centroid of slice 800 (1.6 msec into pulse) versus distance for the commissioning geometry. The red line is for the horizontal centroid and the blue line is for the vertical centroid. 


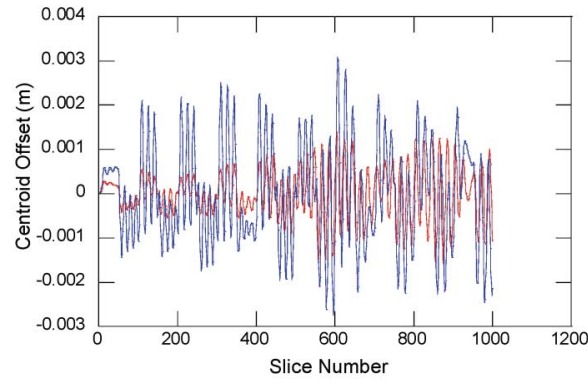

(a)

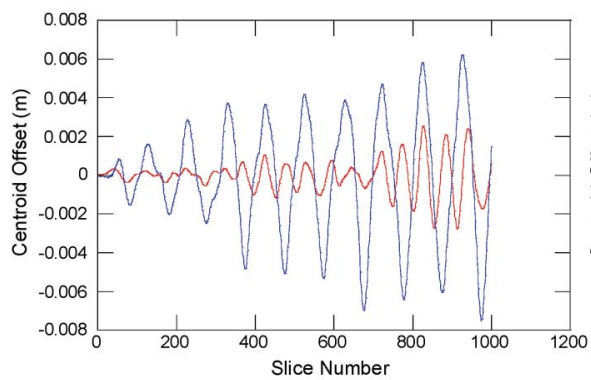

(c)

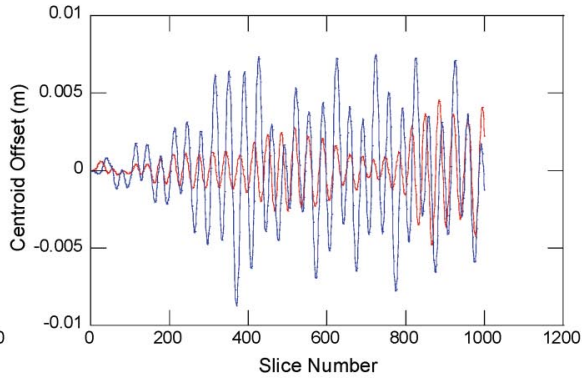

(b)

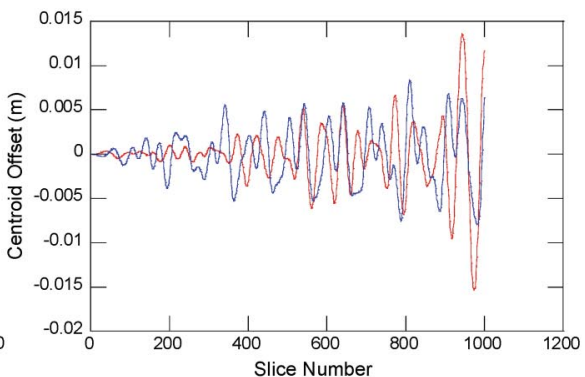

(d)

FIG. 33. (Color) Ion centroids at (a) $2.5 \mathrm{~m}$, (b) $5 \mathrm{~m}$, (c) $7.5 \mathrm{~m}$, and (d) $10 \mathrm{~m}$ for a $2 \mathrm{msec}$ beam in the downstream transport, for an initial offset period of $200 \mathrm{nsec}$. The red line is for the horizontal offset and the blue line is for the vertical offset.

Comparing this case with the one for the commissioning geometry, we find that the instability at the higher energy beam has a larger growth rate. This is surprising, considering the higher energy beam has a larger inertia and that the large-scale changing of the beam radius must be detuning the instability somewhat. We can conclude that the closely spaced periodic focusing in the commissioning geometry still provides some suppression of the ion-hose

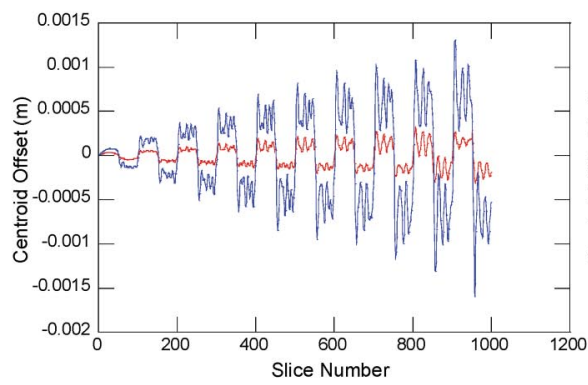

(a)

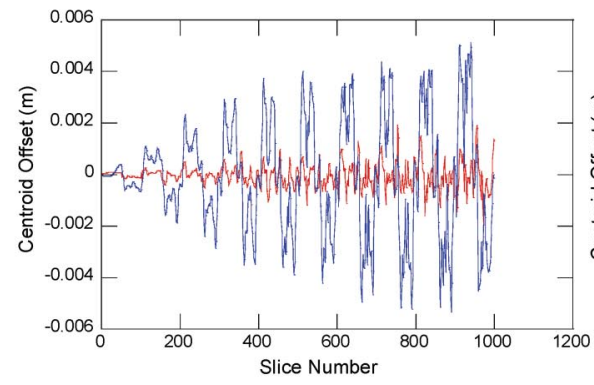

(c)

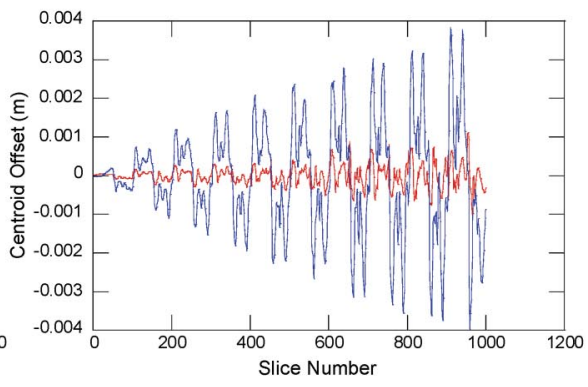

(b)

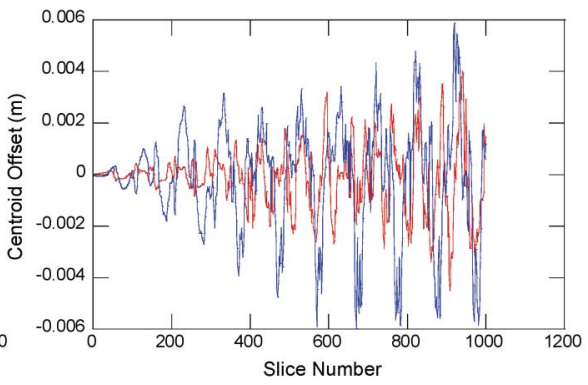

(d)

FIG. 34. (Color) Electron centroids at (a) $2.5 \mathrm{~m}$, (b) $5 \mathrm{~m}$, (c) $7.5 \mathrm{~m}$, and (d) $10 \mathrm{~m}$ for a $2 \mathrm{msec}$ beam in the downstream transport, for an initial offset period of $200 \mathrm{nsec}$. The red line is for the horizontal offset and the blue line is for the vertical offset. 
instability that is absent in the downstream configuration. It is important to point out that a focusing tune with larger beam sizes will suppress the instability growth amplitude to an acceptable level.

\section{Discussion}

With these simulations, we have demonstrated modeling of the ion-hose instability for actual accelerator configurations, over sizable distances (over $10 \mathrm{~m}$ ) with relatively fine step sizes (on the order of millimeters), including details of acceleration and external focusing. We have used the hybrid-fluid model to phenomologically describe the onset and development of the instability in regions of interest in the DARHT long-pulse accelerator.

Continuous, uniform focusing will tend to suppress the growth of the instability, by restricting the amplitude of the electron motion. The ion centroid amplitude is tied to the electron motion, because the ions fall out of resonance once the ions leave the potential well of the electrons and the external field restricts the ability of the electron slices to follow the collective ion motion. If the focusing is not continuous, but the gaps between solenoids are small, narrow resonances appear. These narrow resonances probably depends on both the rms focusing strength and its first derivative, and the resonances can be moved around by changing the focusing. Periodic focusing does not restrict as much the electron tendency to follow, and thus drive, the collective ion motion, and very large-amplitude motion can result. If few solenoids are used with long distances in between, wider resonance appear, with greatly increased growth rates. These wide resonances can be found by the electrons either by direct amplification or by harmonic amplification. The amplitude of the centroid oscillations may be augmented by overfocusing from the solenoids, with excessive zero-current phase advance between the solenoids. For this case, the electron centroid motion will tend to follow the local ion resonances, and the growth rate and saturated amplitude will be relatively insensitive to both tuning and beam initial conditions. Use of multiple ion species may lead to some damping.

\section{ACKNOWLEDGMENTS}

This work was supported by funds from the DARHT project at the Los Alamos National Laboratory, operated by the University of California for the U.S. Department of Energy.
[1] R. J. Briggs (private communication).

[2] G. J. Caporaso and J. F. McCarrick, in Proceedings of the 2000 LINAC Conference, Monterey, CA, edited by Alexander Chao (SLAC Report No. SLAC-R-5671, 2000).

[3] T. P. Hughes and T. C. Genomi, in Proceedings of the 2001 Particle Accelerator Conference, Chicago, IL, 2001, IEEE Catalog No. 01CH37268, 2001.

[4] E. P. Lee, Phys. Fluids 21, 1327 (1978).

[5] W. M. Sharp, M. Lampe, and H. S. Uhm, Phys. Fluids 25, 1456 (1982).

[6] H. L. Buchanan, Phys. Fluids 30, 221 (1987).

[7] T. C. Genomi and T.P. Hughes, Phys. Rev. A 46, 5174 (1992).

[8] K. T. Nguyen, R. F. Schneider, J. R. Smith, and H. S. Uhm, Appl. Phys. Lett. 50, 239 (1987).

[9] K. J. O’Brien, G. W. Kamin, T. R. Lockner, J. S. Wagner, I. R. Shokair, P. D. Kiekel, I. Molina, D. J. Armistead, S. Hogeland, E. T. Powell, and R. J. Lipinski, Phys. Rev. Lett. 60, 1278 (1988).

[10] R. J. Lipinski, J. R. Smith, I. R. Shokair, K. W. Stuve, P. Werner, D. J. Armistead, P. D. Kiekel, I. Molina, and S. Hogeland, Phys. Fluids B 2, 2764 (1990).

[11] T. O. Raubenheimer and F. Zimmermann, Phys. Rev. E 52, 5487 (1995).

[12] G. V. Stupakov, T. O. Raubenheimer, and F. Zimmermann, Phys. Rev. E 52, 5499 (1995).

[13] D. Neuffer, E. Colton, G. Swain, H. Thiessen, B. Blind, R. Hardekopf, A. Jason, G. Lawrence, R. Shafer, T. Hardek, J. Hurd, R. Macek, and M. Plum, Part. Accel. 23, 133 (1988).

[14] D. Neuffer, E. Colton, D. Fitzgerald, T. Hardek, R. Hutson, R. Macek, M. Plum, H. Thiessen, and T.-S. Wang, Nucl. Instrum. Methods Phys. Res., Sect. A 321, 1 (1992).

[15] C. Ekdahl et al., IEEE Trans. Plasma Sci. 33, 892 (2005).

[16] M. E. Jones, B. E. Carlsten, M. J. Schmitt, C. A. Aldrich, and E. L. Lindman, Nucl. Instrum. Methods Phys. Res., Sect. A 318, 323 (1992).

[17] I. R. Shokair and J.S. Wagner, Sandia National Laboratories Report No. SAND87-2015, 1987.

[18] T. P. Hughes (private communication).

[19] B. E. Carlsten, Phys. Rev. E 64, 046501 (2001).

[20] F. F. Chen, Introduction to Plasma Physics and Controlled Fusion (Plenum Press, New York, 1988).

[21] B. E. Carlsten, Phys. Plasmas 5, 1148 (1998).

[22] S. Humphries, Jr., Charged Particle Beams (John Wiley and Sons, Inc., New York, 1990).

[23] H. C. Straub, P. Renault, B. G. Lindsay, K. A. Smith, and R. F. Stebbings, Phys. Rev. A 52, 1115 (1995).

[24] K. J. O’Brien, J. Appl. Phys. 65, 9 (1989).

[25] B. E. Carlsten, Phys. Plasmas 6, 3615 (1999). 